ARTICLE

https://doi.org/10.1038/s41467-019-14265-0

\title{
A common wild rice-derived $B O C 1$ allele reduces callus browning in indica rice transformation
}

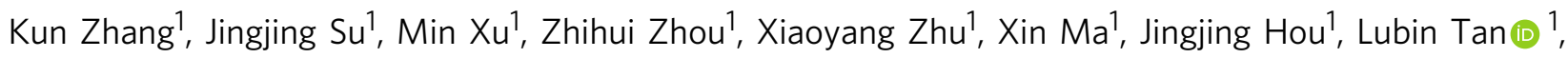

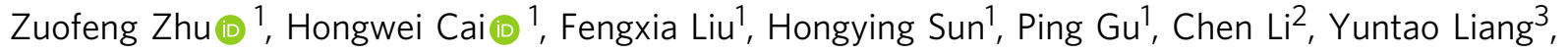
Wensheng Zhao ${ }^{4}$, Chuanqing Sun ${ }^{1,5} \&$ Yongcai Fu+ ${ }^{1 *}$

Callus browning, a common trait derived from the indica rice cultivar (Oryza sativa L.), is a challenge to transformation regeneration. Here, we report the map-based cloning of BROWNING OF CALLUS1 (BOC1) using a population derived from crossing Teqing, an elite indica subspecies exhibiting callus browning, and Yuanjiang, a common wild rice accession (Oryza rufipogon Griff.) that is less susceptible to callus browning. We show that BOC1 encodes a SIMILAR TO RADICAL-INDUCED CELL DEATH ONE (SRO) protein. Callus browning can be reduced by appropriate upregulation of $B O C 1$, which consequently improves the genetic transformation efficiency. The presence of a Tourist-like miniature inverted-repeat transposable element (Tourist MITE) specific to wild rice in the promoter of $B O C 1$ increases the expression of $B O C 1$ in callus. $B O C 1$ may decrease cell senescence and death caused by oxidative stress. Our study provides a gene target for improving tissue culturability and genetic transformation.

\footnotetext{
${ }^{1}$ MOE Key Laboratory of Crop Heterosis and Utilization, National Center for Evaluation of Agricultural Wild Plants (Rice), Beijing Key Laboratory of Crop Genetic Improvement, Department of Plant Genetics and Breeding, China Agricultural University, Beijing 100193, China. ${ }^{2}$ Rice Research Institute, Guangdong Academy of Agricultural Sciences, Guangzhou 510640, China. ${ }^{3}$ Rice Research Institute, Guangxi Academy of Agricultural Sciences, Nanning 530007, China. ${ }^{4}$ State Key Laboratory of Agrobiotechnology and MOA Key Lab of Pest Monitoring and Green Management, China Agricultural University, Beijing 100193, China. ${ }^{5}$ State Key Laboratory of Plant Physiology and Biochemistry, China Agricultural University, Beijing 10093, China. *email: yongcaifu@cau.edu.cn
} 
$\mathrm{n}$ contrast to japonica rice (Oryza sativa ssp. japonica), Agrobacterium tumefaciens-mediated transformation of indica rice (Oryza sativa ssp. indica) is hindered by callus browning. Callus browning is a common feature of many plant species that presents problems for in vitro culture, a process required for transgenic breeding, resulting in decreased regenerative ability, poor growth, and even death ${ }^{1}$. The use of antioxidants ${ }^{2-4}$, adsorbing agents ${ }^{5}$, low salt concentrations ${ }^{6}$, and growth regulators ${ }^{7}$ may lessen the effects of callus browning to a certain degree, but because some species do not tolerate these treatments, there is no universal solution to this problem.

The physiological and biochemical mechanisms of callus browning have been extensively studied. For instance, reducing the activity of polyphenol oxidase (PPO), which catalyzes the oxidation of phenols to quinones that in turn polymerize to form brown pigments ${ }^{8}$, can mitigate the browning of callus ${ }^{9}$. Browning calli have lower antioxidant enzyme activity than non-browning calli and oxidative stress is associated with callus browning ${ }^{10,11}$. Endogenous $^{12}$ and exogenous ${ }^{13}$ ethylene (ET) cause browning in plant cell cultures. In addition, reduced nitrite reductase activity leads to callus browning ${ }^{14}$.

Different periods of in vitro rice tissue culture are controlled by many complex genes, respectively ${ }^{15}$. Several quantitative trait loci (QTLs) for culturability traits in rice have been identified ${ }^{16-24}$. Using positional cloning, a major QTL gene encoding ferredoxinnitrite reductase $(\mathrm{NiR})$ that determines regeneration ability in rice was identified ${ }^{19}$. QTLs involved in callus browning have been identified on all 12 rice chromosomes ${ }^{17,21-24}$. However, to our knowledge, there have been no reports on the isolation of genes related to callus browning in any plant species. The molecular mechanisms underlying physiological processes inducing browning in plant cell culture still remain unclear.

Genetic analyses of rice culturability have mainly been performed on cultivated rice, and few studies have employed common wild rice (Oryza rufipogon Griff.). In this study, we isolate and characterize a gene responsible for mitigating callus browning in rice, named BROWNING OF CALLUS1 (BOC1). The BOC1 allele is specific to common wild rice. $B O C 1$ is an allele of a rice homolog of SRO (SIMILAR TO RCD ONE), termed OsSRO1c. The appropriate upregulation of $B O C 1$ significantly reduces callus browning during callus proliferation. BOC1 is upregulated in common wild rice callus due to the presence of the Tourist MITE in the promoter region of $B O C 1$. Additionally, BOC1 improves the genetic transformation efficiency and frequency of Hygromycin (Hyg)-resistant calli following gene transfer. BOC1 may regulate oxidative stress and programmed cell death (PCD), which may repress the callus browning. Our findings not only elucidate the mechanisms of callus browning during tissue culture, but also provide important insights into establishing a suitable genetic transformation system for the molecular breeding of rice.

\section{Results}

Phenotypic analysis of introgression line YIL25. To identify the gene responsible for callus browning in rice, we constructed a set of introgression lines using a Yuanjiang common wild rice (YJCWR, O. rufipogon) accession that is relatively resistant to browning (Fig. 1b) as a donor and an elite indica cultivar Teqing (O. sativa) that is more susceptible to callus browning (Fig. 1a) as the recipient. We screened the introgression line YIL25 by inoculating mature seeds on unimproved NB (UINB) medium (NB basal medium without additives) for 7 days, and transferred the resulting scutellum-derived calli to UINB medium for 3 weeks of subculture (Fig. 1c). The callus browning rate (CBR) and callus browning index (CBI) were lower in YIL25 than Teqing (Fig. 1j, k). YIL25 contained three YJCWR chromosomal segments on the short arm of chromosomes 2, 3, and 5 (Supplementary Fig. 1). We, therefore, chose YIL25 as a reduced-browning line to cross with Teqing. The $\mathrm{F}_{1}$ plants showed similar phenotypes of CBR and CBI to YIL25 (Supplementary Fig. 2). These results indicated that the reduced-browning trait is controlled by a dominant gene. The histological examination indicated that the cell arrangement of YIL25 calli was more even and the cells were smaller and denser compared to Teqing (Fig. 1d, e). Observations using scanning electron microscopy (SEM) revealed that YIL25 calli consisted of many globular nodules (Fig. 1h) and the cells of YIL25 were turgid (Fig. 1i), whereas Teqing calli had fewer globular nodules (Fig. 1f) and the cells of Teqing were flaccid (Fig. 1g).

Map-based cloning of the callus browning trait gene. To clone the gene for the callus browning trait, we constructed a $F_{2}$ population of 198 segregating plants derived from a cross between YIL25 and Teqing. We used the CBI as a phenotypic index as it reflects the frequency and degree of callus browning. First, we measured the CBI on the seed-derived callus of the 198 individual plants. We then performed QTL analysis using simple sequence repeat (SSR) markers. The callus browning trait QTL, qCBT3, which is located between SSR markers RM3131 and RM3766 on the short arm of chromosome 3, had the largest effect, explaining $14 \%$ of the phenotypic variance associated with decreased CBI (Fig. 2a; Supplementary Table 1).

For high-resolution mapping, we selected $\mathrm{F}_{2}$ individuals that were heterozygous at the qCBT3 locus (RM3131-RM3766), containing one chromosomal segment from Teqing and one from YJCWR by self-crossing to construct a segregating population to eliminate gene interference on introgression segments of chromosomes 2 and 5 (Fig. 2b). We identified plants with recombination events between markers RM3131 and RM3766 by genotyping the 6377 individuals in the population and evaluated the CBI of these recombinant individuals (Fig. 2b). We then developed single nucleotide polymorphism (SNP) markers and delimited $q C B T 3$ to an $18.6 \mathrm{~kb}$ region between SNP markers SN11 and SN13. This region contained only one predicted gene (LOC_OsO3g12820) in the Nipponbare reference genome (The Rice Genome Annotation Project Database, Fig. 2c). Sequence analysis of the coding region in this interval revealed no sequence variation between Teqing and YIL25. Next, we compared the promoter sequences upstream of the translation start site between Teqing and YIL25 and identified one $337 \mathrm{bp}$ deletion, one $1 \mathrm{bp}$ deletion, and three SNPs in Teqing (Fig. 2d; Supplementary Fig. 3). Therefore, we chose LOC_Os03g12820 as the candidate gene for BROWNING OF CALLUS1 (BOC1).

Functional analysis of BOC1. To elucidate the function of LOC_Os03g12820, we constructed an RNA interference construct targeting LOC_Os03g12820 and transformed it into introgression line YIL25 (designated as pRi-BOC1-YIL25). Eighteen transgenic lines were obtained. Reverse transcription-quantitative PCR (RTqPCR) analysis showed that LOC_OsO3g12820 was expressed at lower levels in the $\mathrm{pRi}$-BOC1-YIL25 positive transgenic lines than in the negative (non-transformed) plants (Fig. 3e). In addition, RNAi suppression of LOC_Os03g12820 in the pRi-BOC1-YIL25 positive transgenic lines to the level of Teqing caused serious callus browning compared to the non-transformed controls (Fig. 3a-e).

Furthermore, we introduced a complementation vector containing the promoter, open reading frame and $3^{\prime}$-UTR of LOC_Os03g12820 from YIL25 into Teqing (designated as pCPL-BOC1-Teqing) and obtained 15 positive transgenic plants. 

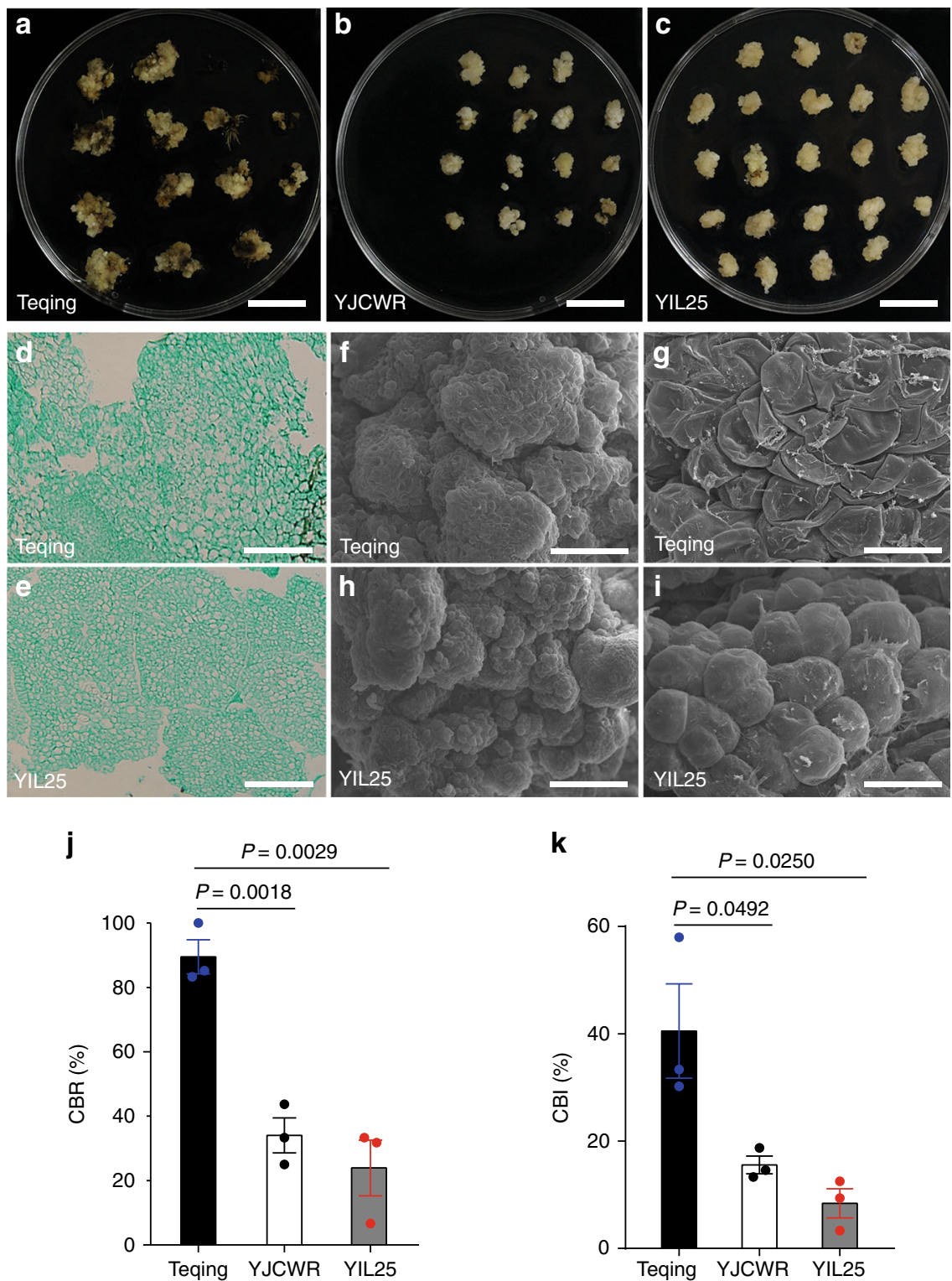

Fig. 1 Phenotypic analysis of calli subcultured for $\mathbf{2 1}$ days. a-c Callus browning phenotypes observed in the calli after subculture for 21 days including a indica variety Teqing, b Yuanjiang wild rice (Oryza rufipogon, YJCWR), and $\mathbf{c}$ the introgression line YIL25. Scale bars, $2 \mathrm{~cm}$. $\mathbf{d}$, e Paraffin sections of $\mathbf{d}$ Teqing and $\mathbf{e}$ YIL25. Scale bars, $100 \mu \mathrm{m}$. f-i Scanning electron microscopy of Teqing and YIL25 calli. f Teqing calli. Thirty calli each from Teqing and YIL25 were collected for analysis of paraffin sections and scanning electron microscopy. Scale bars, $300 \mu \mathrm{m}$. $\mathbf{g}$ Enlarged from (f). Scale bars, $40 \mu \mathrm{m}$. $\mathbf{h}$ YIL25 calli. Scale bars, $300 \mu \mathrm{m}$. i Enlarged from (h). Scale bars, $40 \mu \mathrm{m}$. $\mathbf{j}$, $\mathbf{k}$ Comparison of the CBR and CBI values of various calli. Values in $(\mathbf{k}, \mathbf{I})$ are means \pm SE $(n=3$ biologically independent samples). Two-tailed Student's $t$-tests were performed to determine significant differences. Source data underlying Figs. $\mathbf{j}$, $\mathbf{k}$ are provided as a Source Data file.

RT-qPCR analysis showed that the relative expression levels of LOC_Os03g12820 were significantly elevated in the pCPL-BOC1Teqing positive plants compared to the negative plants (Teqing) (Fig. 3j). The CBI was reduced in these positive lines when the expression level of LOC_Os03g12820 was elevated at least 1.8 fold compared with the negative plants (Fig. $3 \mathrm{f}-\mathrm{j}$ ). This finding indicates that LOC_Os03g12820 mitigates the callus browning phenotype. These results suggest that LOC_Oso3g12820 is BOC1 regulates callus browning in rice.

Next, we generated transgenic Teqing plants overexpressing the LOC_Os03g12820 coding sequence of YIL25 (as shown in Supplementary Fig. 3, the rose red and red underline represent the nucleotide sequence of the overexpression construct, designated as pOE-BOC1-Teqing) and obtained 22 positive transgenic lines. RT-qPCR indicated that $B O C 1$ was strongly upregulated in the pOE-BOC1-Teqing positive transgenic lines compared to the negative plants (Fig. 3o). Nevertheless, when $B O C 1$ expression levels were 8.8-times higher in the tested pOEBOC1-Teqing positive lines compared to the negative plants, callus browning was more severe (Fig. $3 \mathrm{k}-\mathrm{o}$ ). This result indicates that only the appropriate expression of $B O C 1$ reduces callus browning.

BOC1 encodes an SRO protein of 463 amino acids, which includes a putative N-terminal RNA Recognition Motif (RRM) domain, a poly (ADP-ribose) polymerase (PARP)-like ADPribose transferase catalytic domain, and a C-terminal RST (RCD1, [RADICAL-INDUCED CELL DEATH1]-SRO [SIMILAR TO RCD ONE]-TAF4 [TBP-ASSOCIATED FACTOR4] (RST) domain (https://www.ncbi.nlm.nih.gov/Structure/cdd/wrpsb.cgi; Supplementary Fig. 4a). To evaluate the conservation of BOC1 


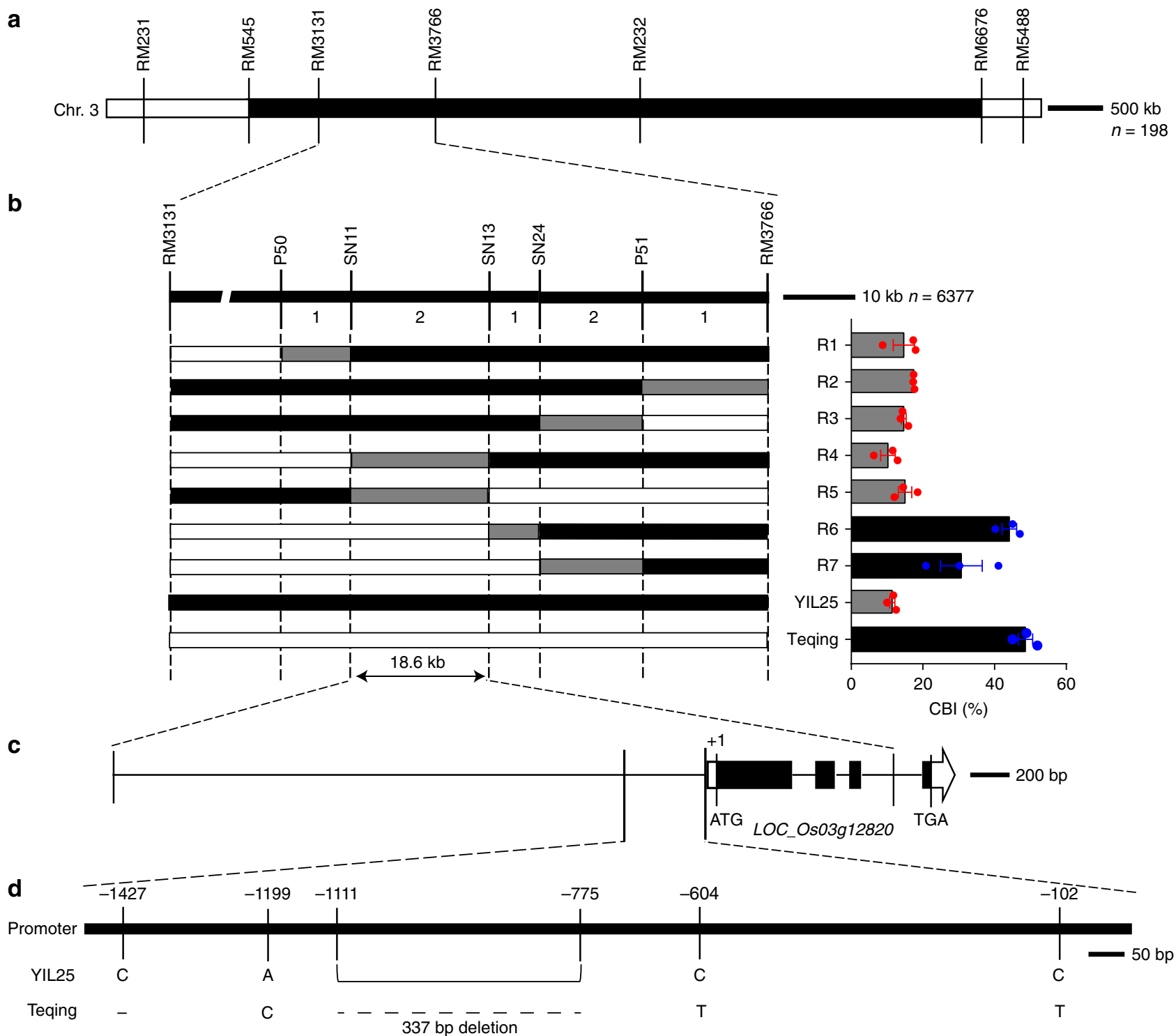

Fig. 2 Map-based cloning of the callus browning trait gene. a qCBT3 was initially localized to a region between markers RM3131 and RM3766 on the short arm of chromosome 3 ( $n=198$ plants of $F_{2}$ populations derived from a cross between YIL25 and Teqing). Scale bar, $500 \mathrm{~kb}$. b Positional cloning narrowed the qCBT3 locus to an $18.6 \mathrm{~kb}$ region between markers SN11 and SN13 ( $n=6377$ individuals used for high-resolution mapping). R1 through R7 are recombinants. The black, white, and gray regions indicate homozygous for the YIL25 genome, homozygous for the Teqing genome, and the interval in the chromosome where crossover took place, respectively. Scale bar, $10 \mathrm{~kb}$. LOC_Os03g12820 is the candidate gene BROWNING OF CALLUS1 (BOC1). Data are means ( $n=3$ biologically independent samples), with error bars showing standard error. c Structure of the candidate gene BOC1. White box, white box with arrow, black boxes, and lines between boxes indicate the $5^{\prime}$-UTR, $3^{\prime}$-UTR, exons, and introns, respectively. d Mutation sites of the BOC1 promoter in YIL25 and Teqing. The ellipsis indicates deletion of bases. Scale bar, 50 bp. Source data underlying Figs. $\mathbf{a}, \mathbf{b}$ are provided as a Source Data file.

in other plant species, we performed protein sequence alignment using the full-length protein sequence of BOC1 as a query in BLAST analysis and selected 24 putative homologs (sharing > $80 \%$ sequence identity with $\mathrm{BOC} 1$ at the nucleotide level) to construct a phylogenetic tree. Phylogenetic analysis indicated that BOC1 is closely related to other monocots, such as Oryza brachyantha, Brachypodium distachyon, Triticum urartu, and Aegilops tauschii (Supplementary Fig. 4b).

Transcriptional characterization of BOC1. To elucidate the expression profiles of $B O C 1$, we analyzed the expression pattern of BOC1 in different organs by RT-qPCR. The expression level of BOC1 was highest in YIL25 calli after 21 days of subculture, followed by the tiller base, roots, culms, and leaves, and it was lowest in young panicles (Supplementary Fig. 5). BOC1 expression increased gradually in Teqing and YIL25 throughout tissue culture. BOC1 expression did not significantly differ between Teqing and YIL25 during the early stage of tissue culture, but significant differences were observed during the later stage; $B O C 1$ expression was notably higher in YIL25 than in Teqing in callus after 21 days of subculture (Fig. 4a). We further examined the expression of BOC1 in calli after 21 days of subculture by RNA in situ hybridization and observed that the hybridization signals were stronger in YIL25 than in Teqing, confirming the notion that BOC1 is expressed at higher levels in YIL25 callus (Fig. 4b).

Sequencing analysis revealed one $337 \mathrm{bp}$ deletion, one $1 \mathrm{bp}$ deletion, and three SNPs in the promoter region of $B O C 1$ between Teqing and YIL25 (Fig. 4c). To determine whether these deletions and SNPs in the BOC1 promoter region directly 

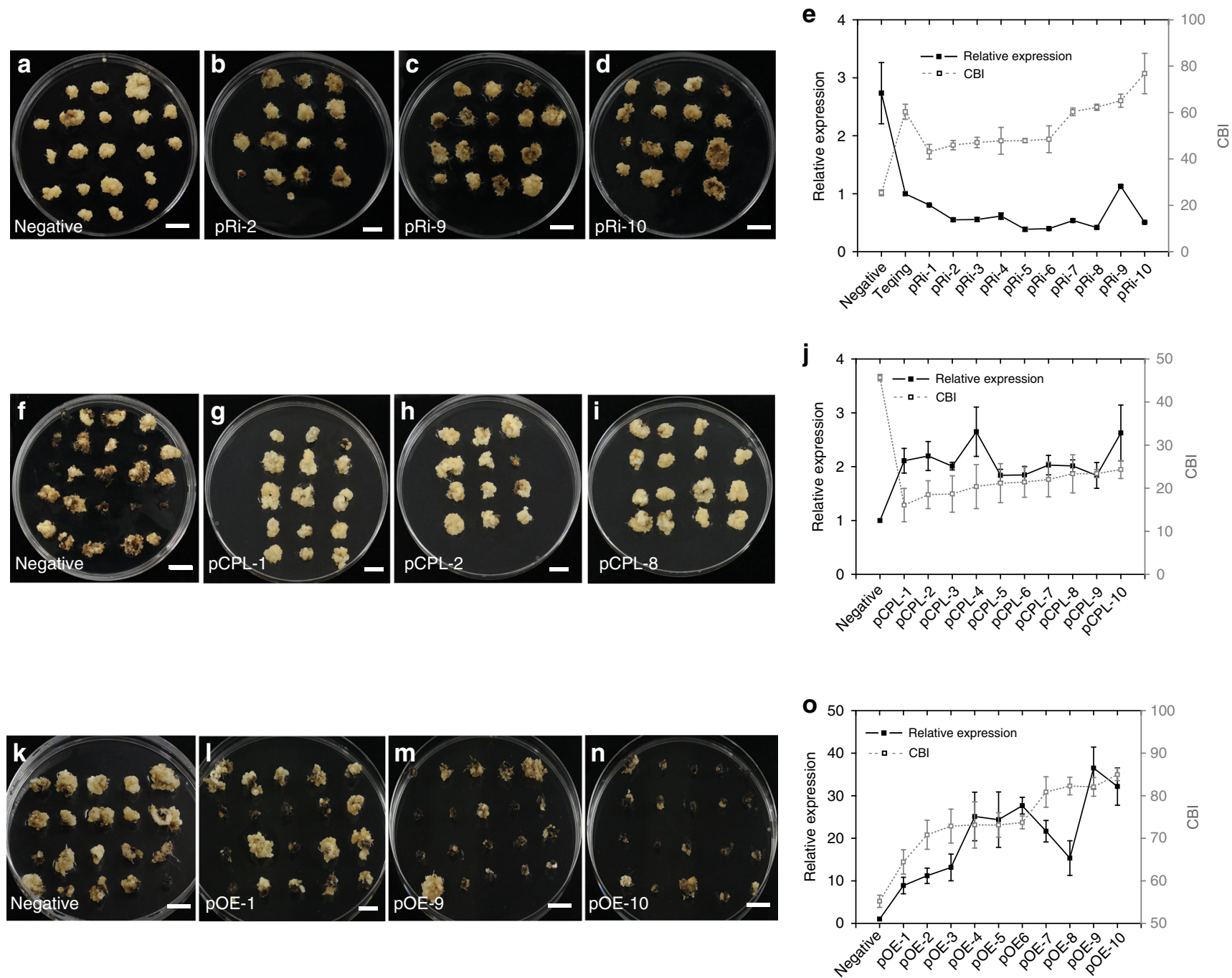

Fig. 3 Functional analysis of BOC1. a-d Phenotypes of calli from pRi-BOC1-YIL25 transgenic plants $(n=18)$. Negative represents segregating plants from the transformation process but without the transgene. Scale bars, $1 \mathrm{~cm}$. e Comparison of the relative expression levels and CBI between a negative transgenic line and 10 RNAi lines ( $n=3$ biological replicates). f-i Phenotypes of calli from pCPL-BOC1-Teqing transgenic plants $(n=15)$. Scale bars, $1 \mathrm{~cm}$. $\mathbf{j}$ Comparison of the relative expression levels and CBI in a negative transgenic line and 10 complementation lines $(n=3$ biological replicates). k-n Phenotypes of calli from pOE-BOC1-Teqing transgenic plants $(n=22)$. o Comparison of the relative expression levels and CBI in a negative transgenic line and 10 overexpression lines ( $n=3$ biological replicates). The black line indicates the trend of the relative expression levels, and the gray line indicates the trend of the CBI. Scale bars, $1 \mathrm{~cm}$. Data are means $\pm \mathrm{SE}$, two-tailed Student's t-tests. Source data underlying Figs. e, j, o are provided as a Source Data file.

contribute to its expression, we generated various constructs by inserting different combinations of mutant promoter fragments of YIL25 into binary vector pGreenII 0800-LUC. We introduced these constructs into rice protoplasts produced from callus that had been cultured for 21 days and examined the expression of the luciferase reporter gene (LUC). As shown in Fig. 4c, no significant differences in activity were observed between the YIL25 promoter fragment and mutated promoter fragments containing three SNPs (constructs M2-M4) and one 1 bp deletion (construct M5). However, the $337 \mathrm{bp}$ deletion (construct M1) significantly decreased the promoter activity of BOC1 to a level similar to that of Teqing (Fig. 4c). These results suggest that the $337 \mathrm{bp}$ deletion is responsible for the reduced expression level of BOC1 in Teqing calli. Using the RepeatMasker Web Server (http://www. repeatmasker.org/cgi-bin/WEBRepeatMasker), we examined the rice transposable element database using the 337 bp sequence as a query and determined that it is the Tourist-like miniature inverted-repeat transposable element (Tourist MITE), which belongs to the PIF/Harbinger superfamily. In addition, using
PLACE (Plant Cis-acting Regulatory DNA Elements; http://www. dna.affrc.go.jp/htdocs/PLACE/), we identified three DOFCOREZM (AAAG, DOF TFBS) ${ }^{25}$, one MYB1AT (TAACCA, MYB TFBS) ${ }^{26}$, one MYCCONSENSUSAT (CAGATG, MYC TFBS) ${ }^{26-28}$, and one ACGTATERD1 (ACGT, ACGT TFBS) ${ }^{28}$ in this sequence; these elements are associated with plant stress responses (Supplementary Fig. 6). Subcellular localization analysis indicated that the BOC1GFP fusion protein was specifically targeted to the nucleus in rice protoplasts (Fig. $4 \mathrm{~d}$ ).

BOC1 influences genetic transformation efficiency. To determine if $B O C 1$ enhances the transformation efficiency of rice due to decreased callus browning, we transformed the binary vector pCAMBIA1300 into YIL25 (YIL25 1300), Teqing (Teqing 1300), and Teqing 1300 as a control. The pCAMBIA1300-BOC1 complementation construct from YIL25 was transformed into Teqing (Teqing pCPL) by Agrobacterium tumefaciens-mediated transformation and cultured on UINB medium for hygromycin (Hyg) 
a

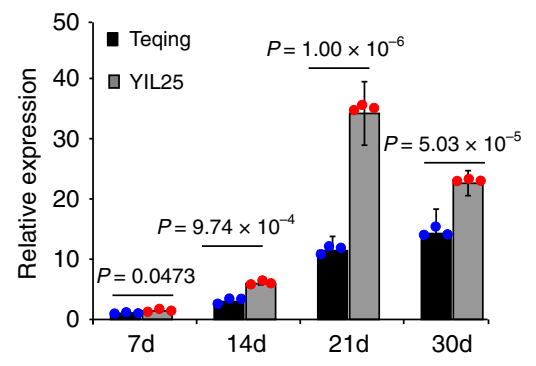

b

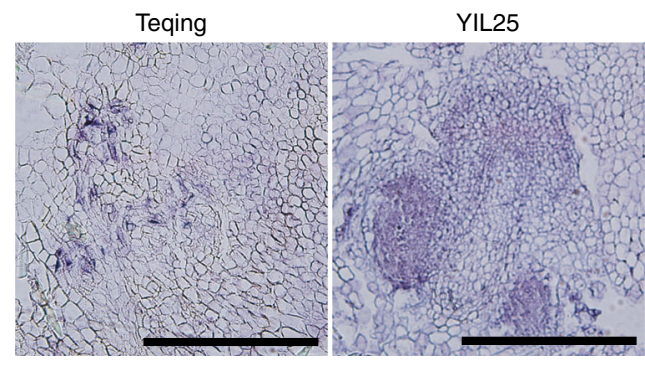

C

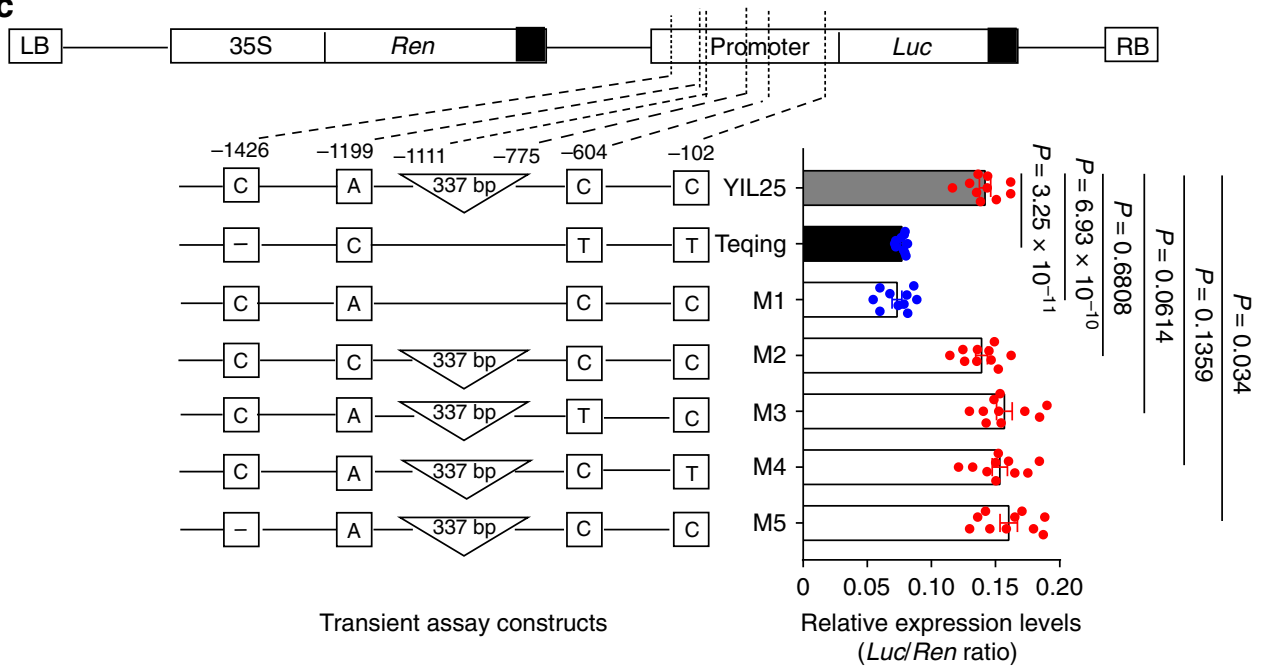

d
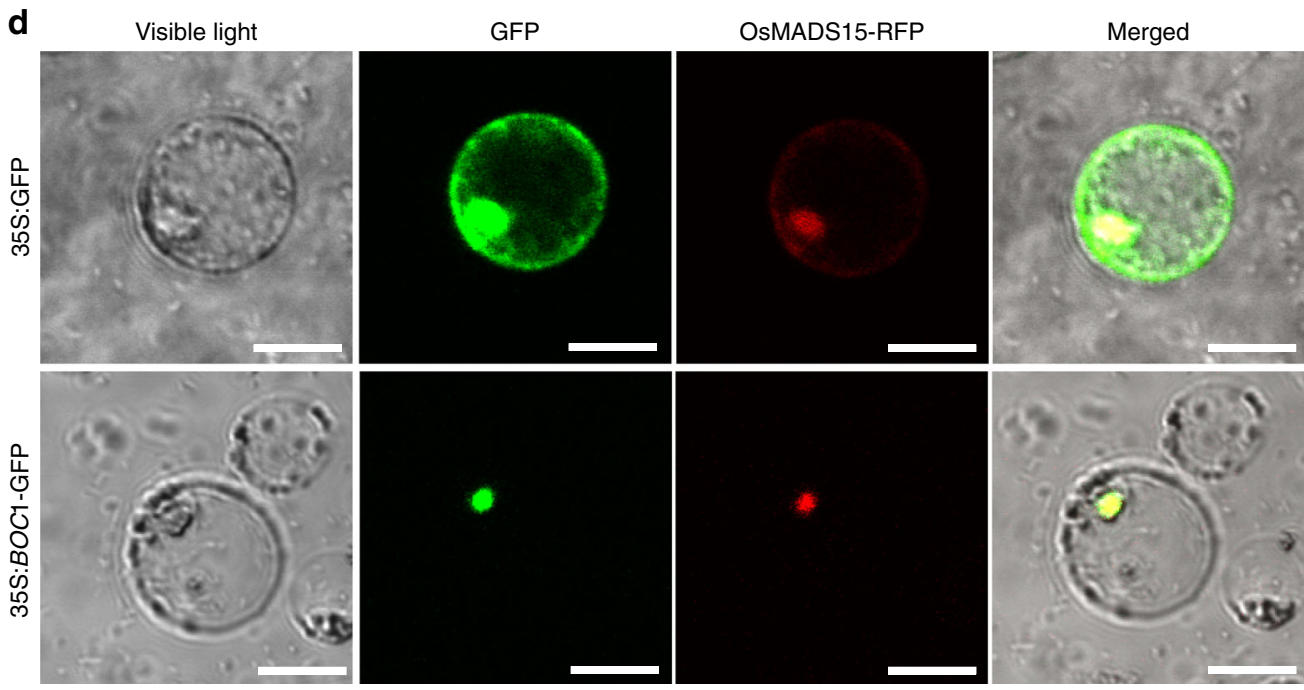

Fig. 4 Transcriptional characterization of BOC1. a Expression analysis of $B O C 1$ in calli at different stages of subculture. Data are means, with bars showing $\mathrm{SE}, n=3$ biological replicates. Two-tailed Student's t-tests were performed to determine significant differences. $\mathbf{b} B O C 1$ expression patterns in Teqing and YIL25 calli subcultured for 21 days, as revealed by mRNA in situ hybridization. Thirty calli each from Teqing and YIL25 were collected for mRNA in situ hybridization. Scale bars, $100 \mu \mathrm{m}$. c Transient expression assays of the effects of InDels and SNPs in the BOC1 promoter. Top, structure of the pGreenll 0800-LUC vector. Left, constructs with site-directed mutagenesis of the BOC1 promoter. LuC, the target promoter controlling the firefly luciferase reporter gene. The Renilla (Ren) luciferase reporter gene was used as an internal control. M1 to M5 indicate the constructs with site-directed mutations at the 337 bp InDel, three SNPs, and $1 \mathrm{bp}$ InDel. Right, expression levels of Luc relative to Ren. Data are presented as mean \pm SE. Horizontal bars show the SE for all constructs ( $n=10$ biological replicates, two-tailed Student's $t$-tests). d Subcellular localization of BOC1 protein in rice protoplast cells. OsMADS15 is a nuclear localization marker. Scale bars, $20 \mu \mathrm{m}$. P-values of (a, c) were calculated with two-tailed Student's $t$-tests. The subcellular localization assays were performed with three biologically independent experiments. Source data underlying Figs. a, c are provided as a Source Data file. 

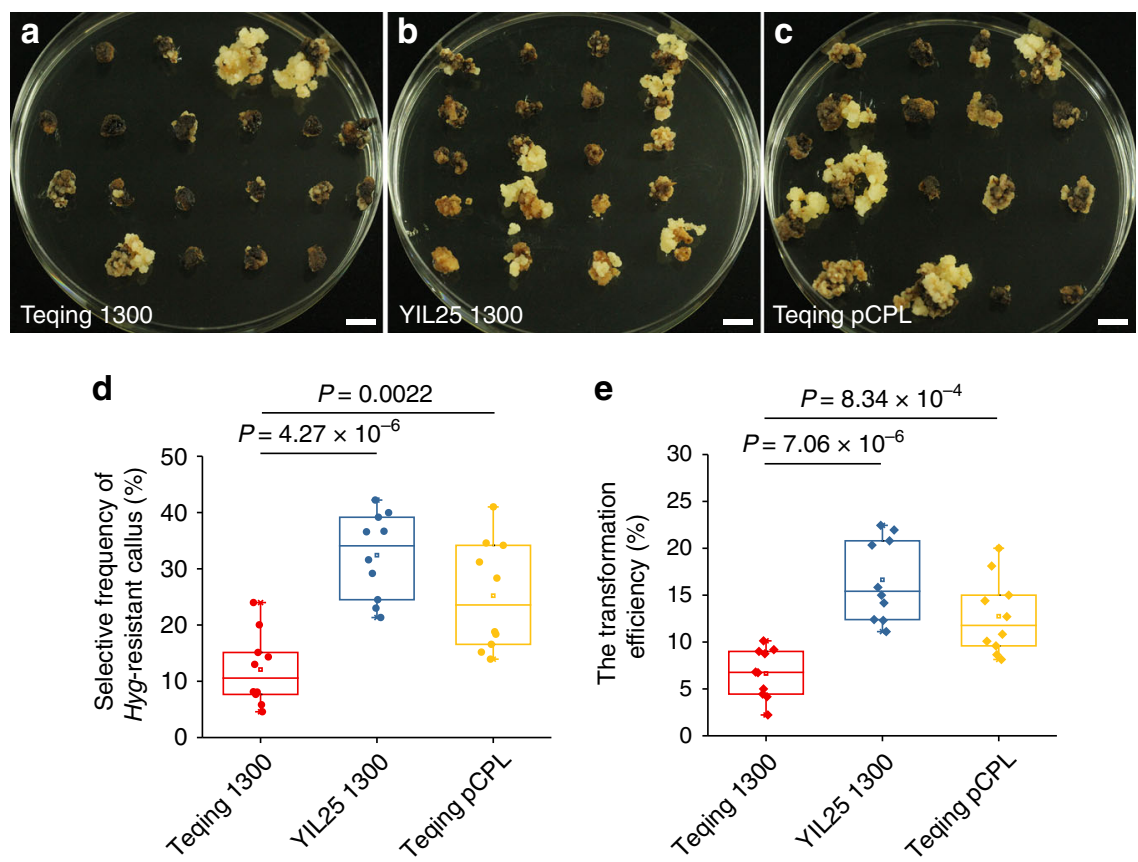

Fig. 5 BOC1 influences genetic transformation efficiency. a-c Transformed calli after three rounds of selection on Hyg-resistant UINB medium. Scale bars, $1 \mathrm{~cm}$. d Comparison of the selection frequency of Hyg-resistant callus. Each dot represents the selective frequency of Hyg-resistant callus of 10 independent experiments. (e) Comparison of the transformation efficiency. Each diamond represents the transformation efficiency of 10 independent experiments. Teqing 1300, Teqing calli were infected with Agrobacterium harboring the binary pCAMBIA1300 empty vector as a control. YIL25 1300, YIL25 calli transformed with the binary vector PCAMBIA1300. Teqing PCPL, Teqing calli infected with Agrobacterium harboring the pCAMBIA1300-BOC1 construct. The hollow box in each column in (d, e) represents the means of the selective frequency of Hyg-resistant callus and the transformation efficiency of 10 replicates, respectively. Box edges represent the 0.25 and 0.75 quantiles, with the median values shown by red, blue and yellow lines, respectively. Whiskers extend to data no more than 1.5 times the interquartile range, and the remaining data are indicated by dots. $P$-values of (d, e) were determined by two-tailed Student's $t$-tests. Source data are provided as a Source Data file.

selection. The selection frequency of Hyg-resistant calli was more than 2.7-fold higher in YIL25 1300 than in Teqing 1300 (Fig. 5a, $\mathrm{b}, \mathrm{d}$ ), and the transformation efficiency was 2.5 -fold higher in YIL25 1300 than in the Teqing 1300 control (Fig. 5e), indicating that YIL25 containing BOC1 is an excellent receptor material for genetic transformation. Meanwhile, the selection frequency of Hyg-resistant calli was 2.1-fold better (Fig. 5a, c, d) and the transformation efficiency was 1.9 -fold better in Teqing pCPL compared to the Teqing 1300 control (Fig. 5e). These results indicate that $\mathrm{BOCl}$ improves the selection frequency of $\mathrm{Hyg}$ resistant calli and the genetic transformation efficiency of rice by reducing callus browning during Agrobacterium-mediated transformation.

Physiological indices related to oxidative stress. To further explore the molecular mechanisms of callus browning, we measured various physiological and biochemical indices in calli at 21 days of subculture. Compared to Teqing, the $\mathrm{H}_{2} \mathrm{O}_{2}$ contents were lower in both YIL25 and pCPL calli and higher in pOE (pOE-BOC1-Teqing) calli (Fig. 6a; Supplementary Fig. 7a). In addition, we used Noninvasive Microtest Technology (NMT) on rice callus to detect intercellular $\mathrm{H}_{2} \mathrm{O}_{2}$ fluxes. There was a significant efflux of intracellular $\mathrm{H}_{2} \mathrm{O}_{2}$ in YIL25 and pCPL calli, whereas Teqing calli exhibited a significant influx of extracellular $\mathrm{H}_{2} \mathrm{O}_{2}$, suggesting that $\mathrm{H}_{2} \mathrm{O}_{2}$ was expelled from YIL25 and pCPL cells to alleviate celluar damage (Fig. 6b). MDA (malondialdehyde) is a biomarker of lipid peroxidation and an indicator of reactive oxygen species (ROS) production. Compared to Teqing, MDA contents were notably lower in the YIL25 and pCPL lines but higher contents in the pOE lines (Fig. 6c; Supplementary Fig. 7b), reflecting less damage from $\mathrm{H}_{2} \mathrm{O}_{2}$ in YIL25 and PCPL plants. Moreover, catalase (CAT), glutathione reductase (GR), and glutathione S-transferase (GST) activity and antioxidant glutathione (GSH) contents were higher in YIL25 and pCPL vs Teqing callus (Fig. 6d-g). However, GST activity and GSH contents were lower in pOE lines than in Teqing callus although CAT and GR activity did not significantly differ among lines (Supplementary Fig. 7c-f).

Finally, the levels of the plant hormones salicylic acid (SA) and ethylene (ET), as well as the direct ET precursor, 1-aminocyclopropane-1-carboxylic acid (ACC), were significantly lower in YIL25 compared to Teqing (Fig. 6h-j). Collectively, these findings suggest that BOC1 may inhibit PCD caused by oxidative stress, thereby reducing callus browning.

RNA-seq analysis of BOC1. To analyze the molecular function of $B O C 1$, we performed RNA-seq experiments using callus that had been subcultured for 21 days from Teqing and YIL25, Teqing and the complementation line pCPL-BOC1-Teqing (pCPL), and also Teqing and the overexpression line pOE-BOC1-Teqing (pOE). We identified 700 differentially expressed genes (DEGs), including 410 DEGs that were upregulated in YIL25 versus Teqing callus and pCPL versus Teqing callus and downregulated in $\mathrm{pOE}$ versus Teqing callus (UUD, Fig. 7a, Supplementary Data 1). The 290 remaining DEGs were downregulated in YIL25 versus Teqing callus and pCPL versus Teqing callus and upregulated in pOE versus Teqing callus (DDU, fold change $\geq 2, \quad F D R<0.001$ ) (Fig. 7b, Supplementary Data 2).

Among the UUD genes, 98 genes were involved in stress responses and cell death (Fig. 7c). Notably, these included 26 pentatricopeptide repeat protein genes, whose dysfunction leads to ROS accumulation; ${ }^{29,30}$ these genes were upregulated in YIL25 

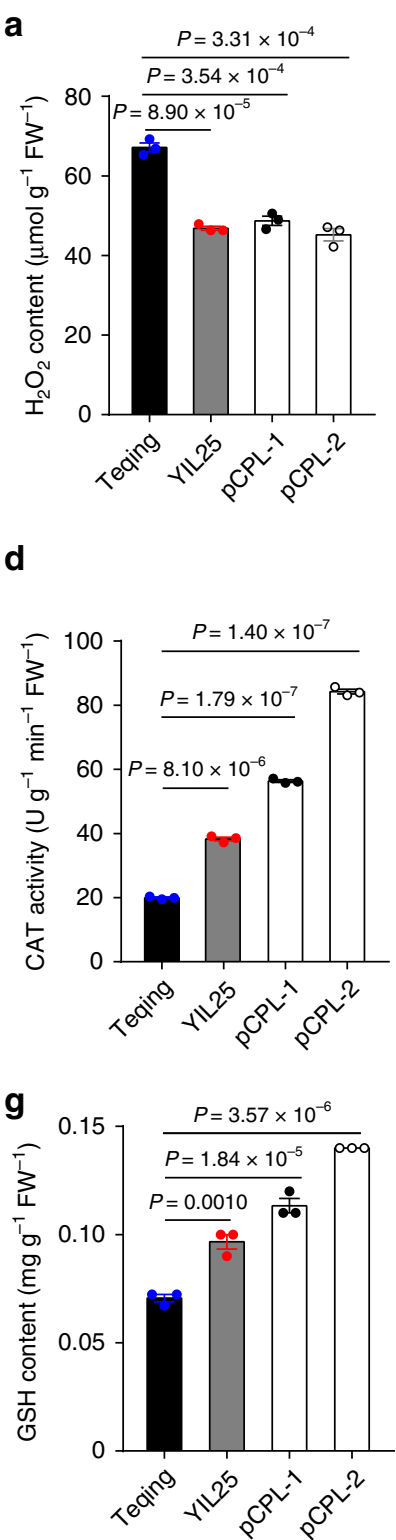

b
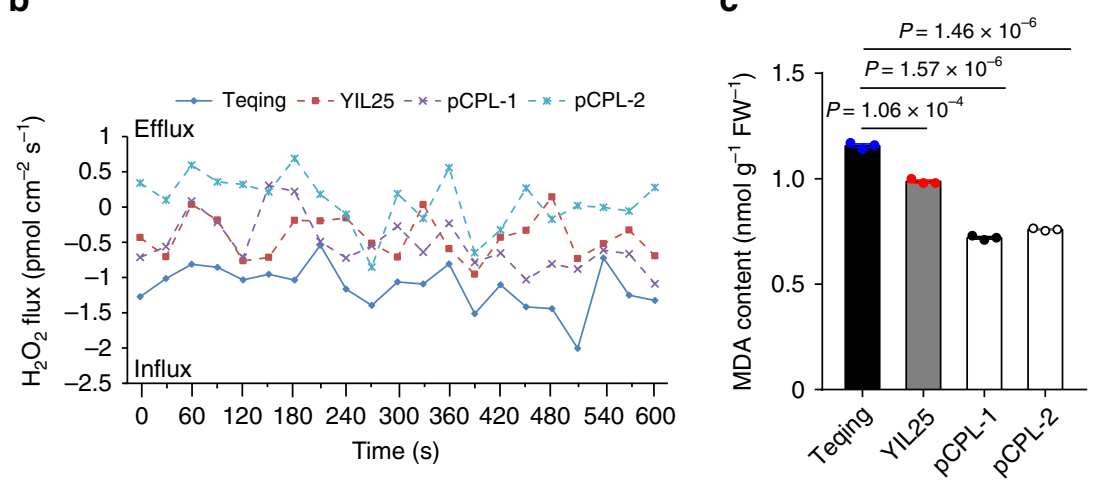

e

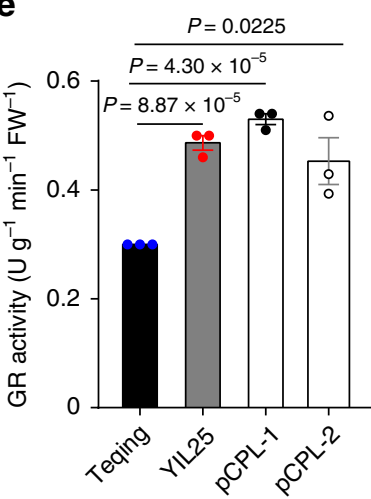

f

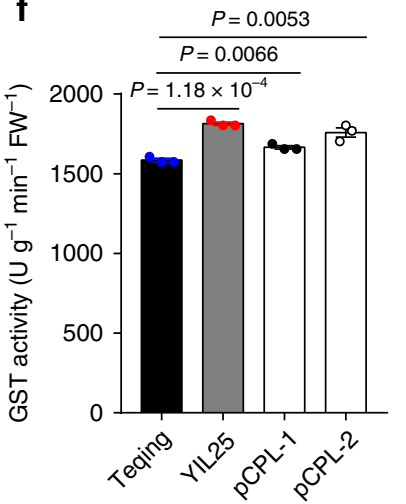

h

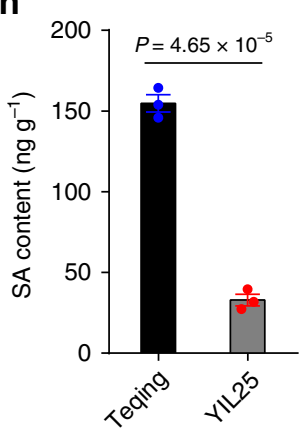

i

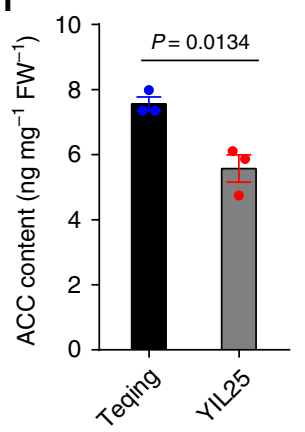

j

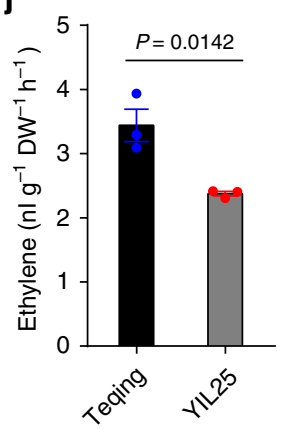

Fig. 6 Physiological and biochemical oxidative stress indices of calli subcultured for $\mathbf{2 1}$ days. a $\mathrm{H}_{2} \mathrm{O}_{2}$ contents in the two parents and two positive complementation transgenic plants. $\mathbf{b} \mathrm{H}_{2} \mathrm{O}_{2}$ flux $(n=4)$. c MDA contents. d-f Assay of antioxidant enzyme activities. CAT, catalase. GR, glutathione reductase. GST, glutathione S-transferase. $\mathbf{g}$ Antioxidant GSH contents. $\mathbf{h}-\mathbf{j}$ Comparison of phytohormone contents between Teqing and YIL25. SA, salicylic acid; ET, ethylene; ACC, 1-aminocyclopropane-1-carboxylic acid, a direct precursor of ET. All values in (a) and (c) to (j) are presented as means \pm SE ( $n=3$ biologically independent samples). P-values were analyzed by two-tailed Student's $t$-tests. Source data are provided as a Source Data file.

and pCPL but downregulated in pOE (Fig. 7c; Supplementary Fig. 8a). GST conjugates with GSH to scavenge ROS in order to protect cellular components from damage. In the current study, 3 GST genes present in the UUD DEG set and absent in the DDU DEG set (Fig. 7c; Supplementary Fig. 8c). In addition, compared to Teqing, GST activity and GSH contents were higher in YIL25 and PCPL callus but lower in pOE callus (Fig. 6f, g; Supplementary Fig. $7 \mathrm{~d}, \mathrm{f})$. Among the UUD genes were four aldehyde dehydrogenase genes; aldehyde dehydrogenase enhances tolerance to various abiotic stresses by scavenging cytotoxic aldehydes (Supplementary Fig. 8d), and MDA contents were higher in of Teqing and pOE callus compared to YIL25 and pCPL callus (Fig. 6c; Supplementary Fig. 7b). LOC_Os01g67980, which negatively regulates the senescence process, encodes senescence-associated protein OsSAG12-1. RNAi transgenic lines declined the expression of OsSAG12-1 and developed early senescence $^{31}$ (Fig. 7c; Supplementary Fig. 8f). In addition, the UUD genes included transcription factor genes associated with stress and defense responses, which might function in maintaining ROS homeostasis (Fig. 7c; Supplementary Fig. 8b, g).

Twelve DDU genes were associated with oxygenase and oxidase; cytochrome P450 has monooxygenase activity as a source of $\operatorname{ROS}^{32}$ (Supplementary Fig. 8h). The DDU group also included genes encoding aspartic proteinases, peptidases, and nuclease (involved in cell degradation), as well as genes encoding polygalacturonase, endoglucanase, and glycosyl hydrolases (involved in cell wall degradation) (Fig. 7c; Supplementary Fig. 8i). Emerging evidence suggests that aspartic proteases are involved in plant development and cell death ${ }^{33}$. Finally, five DDU DEGs encode positive regulators of the senescence process (Fig. 7c). These DEGs include LOC_Os01g53260, encoding WRKY transcription factor OsWRKY23, which positively 
a

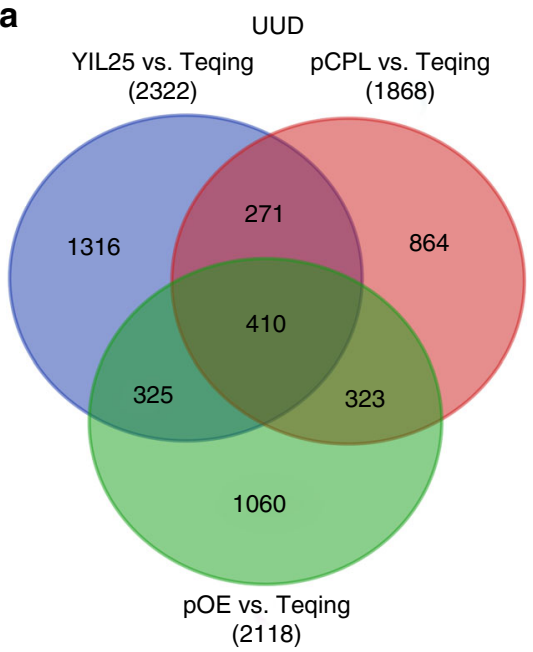

b

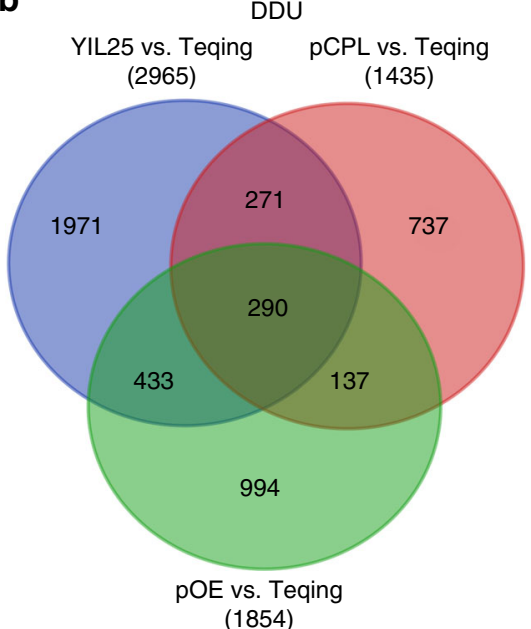

c

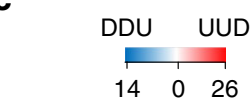

\begin{tabular}{|c|c|c|}
\hline UUD & DDU & Description \\
\hline 26 & 2 & Pentatricopeptide repeat protein \\
\hline 10 & 0 & Transcription factors associated with stress response \\
\hline 3 & 0 & Glutathione S-transferase \\
\hline 4 & 0 & Enzyme of scavenging cytotoxic aldehydes \\
\hline 17 & 4 & Protein kinase \\
\hline 21 & 2 & Other stress and negative regulating cell death related protein \\
\hline 11 & 0 & Defense response \\
\hline 3 & 12 & Oxygenase and oxidase \\
\hline 3 & 14 & Enzymes involved in cell degradation \\
\hline 0 & 5 & Positive regulating cell death \\
\hline
\end{tabular}

Fig. 7 Differentially expressed genes in YIL25, the complementation line, overexpression line, and Teqing revealed by RNA-seq analysis. a Hierarchical clustering of 410 differentially expressed genes (DEGs) upregulated in YIL25 compared to Teqing, DEGs upregulated in the complementation line ( $p C P L$ ) compared to Teqing, and DEGs downregulated in the overexpression line (pOE) compared to Teqing (UUD). b Hierarchical clustering of 290 DEGs downregulated in YIL25 compared to Teqing, DEGs downregulated in PCPL compared to Teqing, and DEGs upregulated in pOE compared to Teqing (DDU). c Hierarchical clustering of DEGs associated with stress response and cell death. The numbers in the boxes represent the number of genes per cluster.

regulates the senescence process $^{34}$, and LOC_Os12g36850, encoding a pathogenesis-related bet $\mathrm{v}$ I family protein similar to probenazole-inducible protein PBZ1, which induces cell death in rice, tobacco, and Arabidopsis via its RNase activity inside the cell $^{35}$ (Supplementary Fig. 8j). When we treated callus that was subcultured for 7 days with $0.5 \% \quad \mathrm{H}_{2} \mathrm{O}_{2}$ for $18 \mathrm{~h}, P B Z$ (LOC_Os12g36850) was upregulated compared to the untreated control (Supplementary Fig. 8k).

Taken together, these findings suggest that the appropriate expression of $B O C 1$ may be beneficial for rice cells to be in reducing state, prevented various stress, and then inhibited cell death.

BOC1 promoter sequence variation affects callus browning. To investigate the relationships between mutations in the BOC1 locus and callus browning, we sequenced a $\sim 4.6 \mathrm{~kb}$ genomic fragment covering the entire $B O C 1$ gene $(2740 \mathrm{bp})$, the $1452 \mathrm{bp}$ $5^{\prime}$-flanking region, and the $380 \mathrm{bp} 3^{\prime}$-flanking region, from $50 \mathrm{O}$. rufipogon accessions (Supplementary Data 3) and 74 Asian rice cultivars (28 japonica and 46 indica cultivars, Supplementary
Data 4). Nucleotide alignment showed that only 19 O. rufipogon accessions contained the Tourist MITE (Supplementary Data 3); however, the element at this locus was absent in all Asian rice cultivars examined (Supplementary Data 4). Therefore, we performed an association test of callus browning after $21 \mathrm{~d}$ of subculture vs. sequence variations in the O. rufipogon accessions and Asian rice cultivars. The association test of the common wild rice revealed the strongest association signals at the Tourist MITE and the four SNPs (Fig. 8a). Furthermore, we investigated the CBI of the 50 accessions of common wild rice and determined that this value was lower in the 19 accessions with the Tourist MITE than in the 31 accessions without this insertion (Fig. 8b). Moreover, the $B O C 1$ gene was expressed at higher levels in the 19 accessions with the Tourist MITE than in the 31 accessions without the Tourist MITE (Fig. 8c; Supplementary Data 3). In addition, we obtained previously reported the genome resequencing data for 446 Oryza rufipogon accessions ${ }^{36}$ and recovered the reads that mapped to the Tourist MITE flanking sequence in these accessions. We detected this site in 226 accessions (48 accessions with this MITE insertion and 178 accessions without this insertion). Forty-one of the 48 accessions with this insertion were in group 


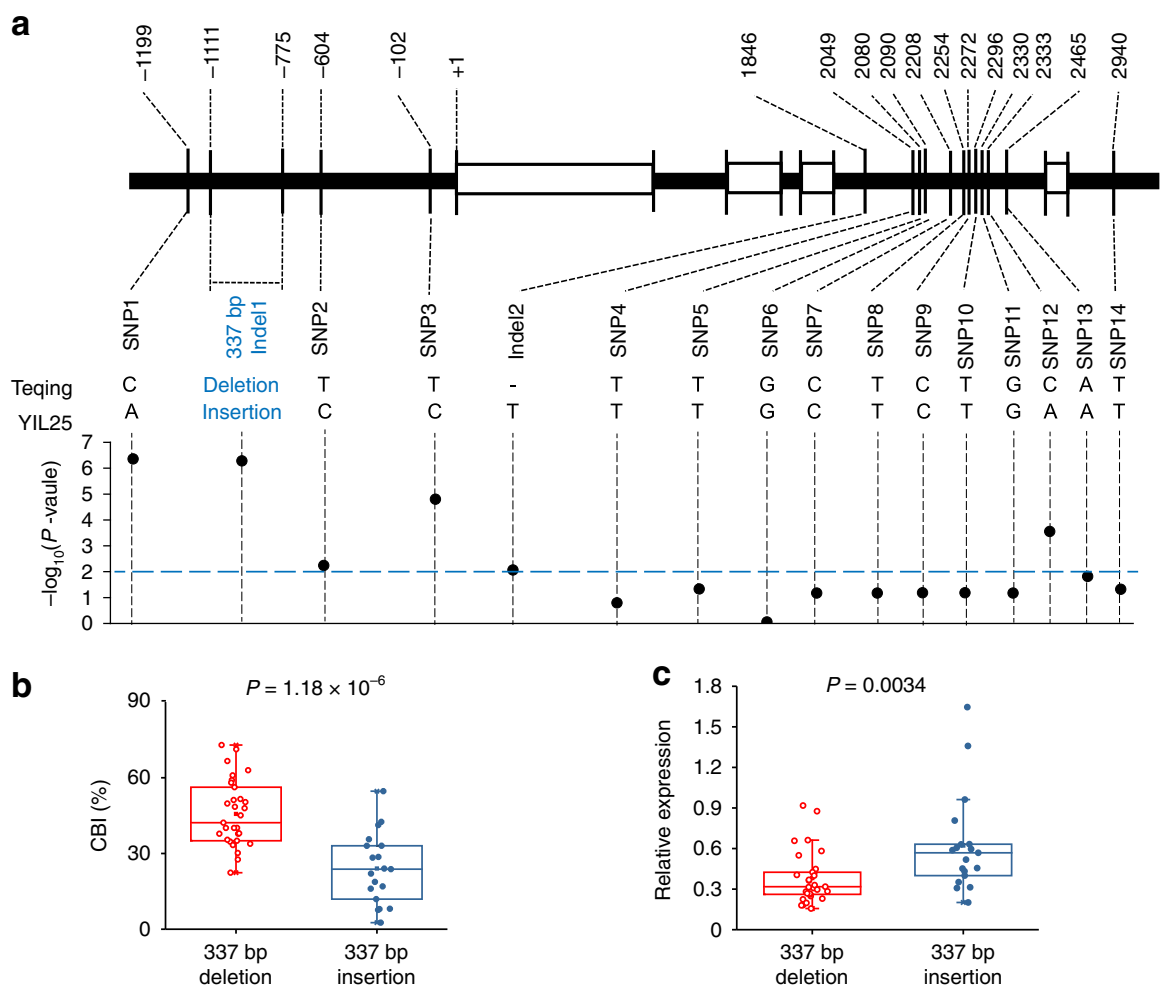

d
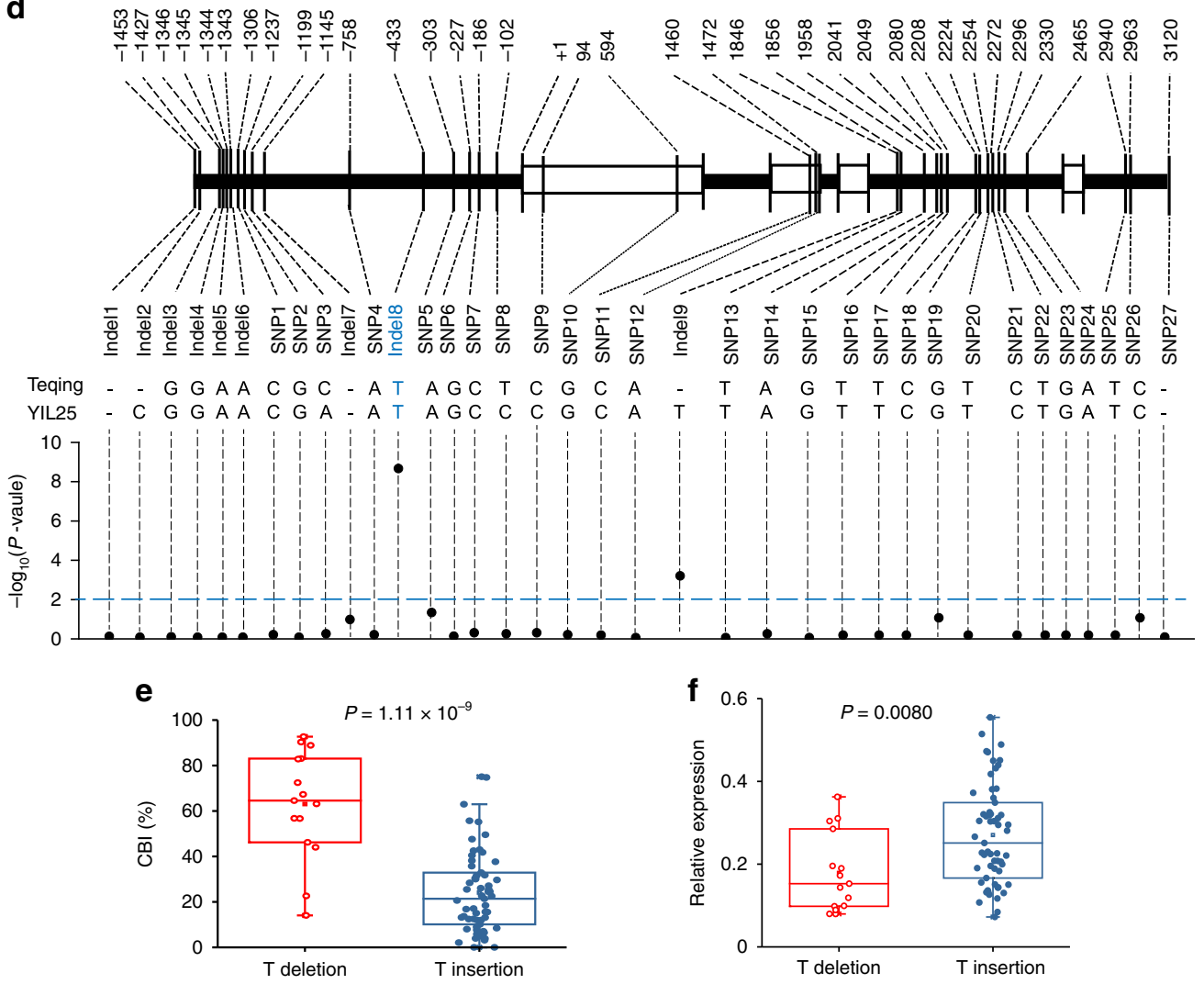

III (closer to japonica) and 7 were in group II (the intermedia group between indica and japonica) (Supplementary Fig. 9a). By contrast, the 178 accessions without this MITE insertion were distributed among all the three groups including 65 in group I (closer to indica), 49 in group II, and 64 in group III (Supplementary Fig. 9a).
Nucleotide alignment of the 74 Asian rice cultivars revealed 9 Indels and 27 SNPs (Fig. 8d; Supplementary Data 4). To ascertain whether BOC1 was responsible for callus browning in Asian rice cultivars, firstly, an association analysis was carried out between callus browning and sequence variations of 74 Asian rice cultivars. The result indicated that the Indel8 $(-433, \mathrm{~T}$ indel) 
Fig. 8 Association test and expression levels of loci with natural variations in BOC1. a Association testing of callus browning in 50 O. rufipogon accessions vs. 16 variant sites in the $4.6 \mathrm{~kb}$ genomic region. Black dots represent 16 variations. b Comparison of CBI in calli subcultured for $21 \mathrm{~d}$ in 31 accessions with the $337 \mathrm{bp}$ deletion vs. 19 accessions with the $337 \mathrm{bp}$ insertion. c Comparison of BOC1 expression in calli subcultured for $21 \mathrm{~d}$ in lines harboring the 337 bp deletion vs. the 337 bp insertion ( $n=3$ biological replicates). d Association testing of callus browning in 74 Asian rice cultivars with 36 variant sites in the $4.6 \mathrm{~kb}$ genomic region. e Comparison of CBI in calli subcultured for $21 \mathrm{~d}$ between 59 accessions with the T deletion and 15 accessions with the $T$ insertion. $\mathbf{f}$ Comparison of $B O C 1$ expression in callus subcultured for $21 \mathrm{~d}$ from rice varieties harboring the $T$ deletion vs. the $T$ insertion ( $n=3$ biological replicates). Box edges of $(\mathbf{b}, \mathbf{c})$ and $(\mathbf{e}, \mathbf{f})$ represent the 0.25 quantile and 0.75 quantile with the median values shown by red and blue lines. Whiskers extend to data no more than 1.5 times the interquartile range, and remaining data are indicated by dots. $P$-values of $(\mathbf{b}, \mathbf{c})$ and $(\mathbf{e}, \mathbf{f})$ were calculated by two-tailed Student's $t$-tests. Source data underlying Figs. b, c, e, f are provided as a Source Data file.

in the promoter of $B O C 1$ was the strongest signals (Fig. 8d). However, this site was no polymorphic in the 50 accessions of $O$. rufipogon (Supplementary Data 3, 4). We further found that the CBI in 15 varieties with $\mathrm{T}$ insertion in the promoter of $B O C 1$ was lower than that in 59 varieties without this insertion (Fig. 8e; Supplementary Data 4). We also analyzed the expression levels in these varieties and the result showed that the expression levels of $B O C 1$ was higher in the 15 varieties with $\mathrm{T}$ insertion in the promoter of $B O C 1$ than that in 59 varieties without this insertion (Fig. 8f; Supplementary Data 4). The above results suggest that the sequence variation in the $B O C 1$ promoter influence callus browning by affecting the expression level of $B O C 1$.

Finally, we analyzed the fixation index $\left(F_{\mathrm{ST}}\right.$, the level of population differentiation), on chromosome 3 in 1083 cultivated indica and japonica rice varieties ${ }^{36}$. The $F_{\mathrm{ST}}$ level in BOC1 was 0.21 , indicating that no significant differences in BOC1 exist between the indica and japonica subspecies (Supplementary Fig. 9b). Therefore, we propose that the effect of $B O C 1$ on callus browning is not related to the differences between the indica and japonica subspecies.

\section{Discussion}

Here, we demonstrated that the proper expression of $B O C 1$ decreases callus browning and increases the transformation efficiency of rice. BOC1 encodes an SRO (SIMILAR TO RCD ONE) protein that is allelic to OsSRO1c, a regulator of oxidative stress responses ${ }^{37}$. RCD1 plays an important role in regulating cell death by controlling oxidative stress responses in Arabidopsis responses $^{38-43}$. OsSRO1c is the major stress-responsive gene in the SRO family. Both ossrolc-1 mutants and amiRNA (artificial microRNA)-OsSRO1c plants are hypersensitive to drought stress at the seedling stage. In addition, OsSRO1c-overexpressing rice plants showed more severe damage under drought stress than wild-type plants, whereas plants overexpressing SNAC1, encoding a NAC transcription factor that directly regulates OsSRO1c expression, showed increased drought resistance ${ }^{29}$. In the current study, RNA interference and $B O C 1$ overexpression plants showed a callus-browning phenotype similar to or more severe than that of Teqing, whereas lines harboring the complementation vector exhibited reduced callus browning, indicating that the appropriate upregulation $B O C 1$ reduces callus browning.

Transposable elements, among the most variable components of the genome, can replicate and integrate into positions, which affects the expression levels of adjacent genes, thereby resulting in genetic diversity ${ }^{44}$. For example, the terminal-repeat retrotransposon in miniature (TRIM) elements insertion in the promoter of MS2 allele in the male-sterile ms 2 mutant resulted in an anther-specific expression pattern in wheat ${ }^{45}$. In maize, an $82 \mathrm{bp}$ miniature inverted-repeat transposable element (MITE) into the promoter of $Z m N A C 111$ represses its expression; this MITE is significantly associated with natural variation ${ }^{46}$. In the current study, the presence of Tourist MITE in the BOC1 promoter significantly increased $B O C 1$ expression in rice callus.
Callus cultured in vitro is subjected to complex growth conditions compared to cells in plants. Callus cells are exposed to the oxygen in air, which tends to result in oxidative stress. Oxidative browning is a prevalent problem in plant tissue culture that can cause cell death, resulting in brown tissues ${ }^{47}$. A moderate increase in the levels of the ROS $\mathrm{H}_{2} \mathrm{O}_{2}$ helps protect plant cells from subsequent exposure to more severe abiotic or oxidative stress ${ }^{48}$. However, the excessive accumulation of $\mathrm{H}_{2} \mathrm{O}_{2}$ might be involved in regulating $\mathrm{PCD}^{38-40,49-51}$. Moreover, high levels of $\mathrm{H}_{2} \mathrm{O}_{2}$ induce the accumulation of SA, resulting in severe oxidative damage ${ }^{39,52}$; in turn, SA pretreatment mediates the accumulation of $\mathrm{H}_{2} \mathrm{O}_{2}{ }^{53}$. Endogenous ET in in vitro culture may inhibit the synthesis of protective enzymes, such as CAT, for scavenging $\mathrm{H}_{2} \mathrm{O}_{2}$, which is involved in senescence, browning, and necrosis of callus $^{54}$. In the current study, YIL25 callus contained decreased levels of $\mathrm{H}_{2} \mathrm{O}_{2}$ and the phytohormones ET and SA, as well as increased levels of CAT, GR, and GST activity compared to Teqing. The RNA-seq data analysis revealed that BOC1 influences the expression of genes associated with ROS production and scavenging, stress response and cell death. In recently report, RCD1 coordinates chloroplast and mitochondrial functions through interaction with transcription factors. RCD1 participates in regulation of mitochondrial respiration and chloroplast ROS processing and may contribute to the survival of plant under a changing environment ${ }^{55}$. However, as shown in Supplementary Fig. 4b, RCD1 and OsSRO1c have different evolutionary histories which may have contrasting functions in the regulation of ROS related processes. In the current study, we use the calli subcultured for $21 \mathrm{~d}$ in the dark and is not necessary for photosynthesis by origin of carbon from medium. Therefore, the interaction and phenotype of BOC1 may be different from seedling stage, which will be an interesting research topic.

Common wild rice, the wild progenitor of cultivated rice, has an important gene pool that includes some alleles no longer present in cultivated rice ${ }^{56}$. The insertion of the $337 \mathrm{bp}$ Tourist MITE in the BOC1 promoter increases BOC1 expression. This insertion is only detected in certain accessions of wild rice, but not in cultivated rice. Therefore, wild rice could be used to provide alleles for improving the tissue culture characteristics and genetic transformation efficiency of cultivated rice. Moreover, the genetic manipulation of the BOC1 promoter in cultivated rice might also improve tissue culture ability. Perhaps the BOC1 allele of wild rice and even similar alleles in other crops of the Gramineae family could be used to create recipient varieties more amenable to genetic transformation. The use of $B O C 1$ in in vitro rice callus propagation might help improve genetic transformation efficiency and selection to facilitate the molecular breeding of rice.

\section{Methods}

Plant materials. The wild rice introgression line YIL25, with reduced levels of callus browning, was derived from a cross between an O. rufipogon accession (YJCWR) as the donor and the elite indica variety Teqing (O. sativa) as the recipient ${ }^{57}$. The mapping population was developed from a cross between introgression line YIL25 and Teqing. The 50 common wild rice accessions and 74 O. sativa 
varieties (28 japonica and 46 indica varieties) used in this study are listed in Supplementary Data 3 and Supplementary Data 4

Tissue culture procedure. Mature, healthy, dehusked seeds were sterilized by immersion in $70 \%$ ethanol for $\sim 2 \mathrm{~min}$, followed by $15 \%$ sodium hypochlorite solution for $15 \mathrm{~min}$ with shaking, and rinsed three or four times with sterile water on an ultraclean workbench. Unimproved NB (UINB) medium (NB medium containing N6 macronutrient components ${ }^{58}$, B5 micronutrient components, and organic components ${ }^{59}$, supplemented with $2 \mathrm{mg} / \mathrm{L}$ of $2,4-\mathrm{D}$ and $30 \mathrm{~g} / \mathrm{L}$ of sucrose) was used for callus induction and subculture. The $\mathrm{pH}$ of the medium was adjusted to 5.8 with $1 \mathrm{~N} \mathrm{KOH}$, and $3 \mathrm{~g} / \mathrm{L}$ of Phytagel was added before autoclaving at $121^{\circ} \mathrm{C}$ for $20 \mathrm{~min}$. Approximately 90 mature seeds per line, evenly distributed among three Petri dishes, were incubated in induction medium for 7 days at $28^{\circ} \mathrm{C}$ in the dark. The induced calli were transferred to subculture (UINB) medium and incubated in the dark at $28^{\circ} \mathrm{C}$ for 3 weeks. The traits of the calli in each Petri dish were recorded.

Phenotype observations. The tendency for callus browning was categorized into four levels: (0) less than 1/10 of the callus tissue was brown (recorded as no browning); (1) $1 / 10 \sim 1 / 3$ of the callus tissue was brown, considered light browning; (2) $1 / 3 \sim 2 / 3$ of the callus tissue was brown, considered medium browning; (3) $2 / 3$ $\sim 1$ of the callus tissue was brown, considered deep browning; and (4) the callus was completely brown. The tendency for callus browning, selection frequency of Hyg-resistant calli and transformation efficiency were calculated according to the following Eqs. (1, 2, 3, and 4):

Callus browning rate $(\mathrm{CBR})=$ (number of calli showing browning/ number of transferred calli) $\times 100 \%$

Callus browning index $(\mathrm{CBI})=(\Sigma$ number of calli at each browning level $\times$ browning level $) /$ (number of transferred calli $\times$ highest browning level) $\times 100 \%$

Selection frequency of $\mathrm{Hyg}$-resistant calli $=$ (number of $\mathrm{Hyg}$-resistant calli/ number of infected calli) $\times 100 \%$

Transformation efficiency $=$ (number of independent transgenic events regenerating plants $/$ number of transferred calli) $\times 100 \%$

Scanning electron microscopy. To prepare histological sections, calli that had been subcultured for 21 days were fixed in $2.5 \%$ glutaraldehyde-phosphate buffer saline fixative solution ( $\mathrm{pH}$ 7.2) for more than $2 \mathrm{~h}$ and postfixed in the same buffer containing $1 \% \mathrm{OsO}_{4}$. After being dehydrated through an ethanol series and dried using a carbon dioxide critical-point dryer, the calli were cleaned with ethanol and dried at $45^{\circ} \mathrm{C}$. The dry calli were gold plated and photographed under a Hitachi S3400 scanning electron microscope (Japan).

Primers. The primers used in this study are listed in Supplementary Data 5.

Genetic confirmation. The RNA interference construct was generated by inserting a hairpin sequence with a $350 \mathrm{bp}$ and a $346 \mathrm{bp}$ cDNA inverted repeat fragment of BOC1 into the pTCK303 vector driven by the maize Ubiquitin promoter and transformed into YIL25 (pRi-BOC1-YIL25). A 4572 bp genomic fragment from YIL25 harboring the entire BOC1 gene sequence with the 1452 bp $5^{\prime}$-flanking region and the 380 bp $3^{\prime}$-flanking region was amplified and inserted into binary vector pCAMBIA1300 (http://www.cambia.org) to form the complementary con struct (pCPL). The overexpression vector (pOE) contained the 1392 bp YIL25 BOC1-ORF, which was amplified and inserted into the pCAMBIA1301 vector (http://www.cambia.org) driven by the Ubiquitin promoter. Both the pCPL and pOE constructs were transferred into Teqing and designated as pCPL-BOC1Teqing and pOE-BOC1-Teqing, respectively. Relevant primer sequences are listed in Supplementary Data 5.

RT-qPCR. Total RNA was extracted from various samples using TRIzol reagent (Life Technologies) and purified using an RNeasy Mini Kit (Qiagen) following the manufacturer's instructions. First-strand cDNA was synthesized using Oligo $(\mathrm{dT})_{15}$ primers (TaKaRa) and SuperScript III Reverse Transcriptase (Invitrogen) with $3 \mu \mathrm{g}$ of total RNA. The qPCR was performed using a CFX96 Real-Time System (Bio$\mathrm{Rad}$ ), with each reaction containing $5 \mathrm{ng}$ of first-strand cDNAs, $4 \mu \mathrm{M}$ of genespecific primers, and $5 \mu \mathrm{L}$ of real-time PCR SYBR MIX (iQ ${ }^{\mathrm{m}}$ SYBR $^{\circledR}$ Green Supermix, Bio-Rad) under the following conditions: initial denaturation at $95^{\circ} \mathrm{C}$ for $3 \mathrm{~min}$ followed by $40 \mathrm{cycles}$ of $95^{\circ} \mathrm{C}$ for $30 \mathrm{~s}, 58^{\circ} \mathrm{C}$ for $30 \mathrm{~s}$, and $72{ }^{\circ} \mathrm{C}$ for $30 \mathrm{~s}$. The rice housekeeping gene Actin (LOC_Os03g50885) was used as an internal control to normalize the gene expression data using the relative quantification method $\left(2^{-\Delta \Delta C T}\right)^{60}$. Each set of experiments was repeated three times. The primers used for RT-qPCR and cDNA amplification are listed in Supplementary Data 5.
mRNA in situ hybridization. mRNA in situ hybridization was performed with the method of Javelle et al. ${ }^{61}$ with minor modifications. YIL25 and Teqing calli that had been subcultured for 21 days were fixed in $3.7 \%$ FAA solution at $4{ }^{\circ} \mathrm{C}$ overnight, dehydrated, and embedded in paraffin (Paraplast Plus, Fisher Scientific). The calli were sliced into $10-\mu \mathrm{m}$ sections with a microtome (Leica RM2145). A 344 bp fragment of BOC1 CDNA was amplified and used as the template to generate sense and antisense RNA probes, which were labeled using a DIG RNA labeling Kit (Roche) according to the manufacturer's instructions. The hybridized slides were observed under a microscope (Leica DMR). Relevant primer sequences are listed in Supplementary Data 5.

Luciferase assays. The promoter sequences upstream of the BOC1 translation start site of YIL25 and Teqing were amplified using specific primers designed with site-directed mutations at the InDels and SNPs. The fragments were inserted into the pGreenII 0800-LUC vector to drive the expression of the firefly luciferase (LUC) gene. The effector plasmid DNA was transformed into rice protoplasts prepared from rice calli that had been subcultured for 21 days in the dark using the polyethylene glycol-mediated method ${ }^{62}$. The enzyme solution contained the following: $1.5 \%$ (w/v) "Onozuka" R-10 cellulose (Yakult Pharmaceutical Industry), 0.75\% (w/ v) macerozyme R-10 (Yakult Pharmaceutical Industry), 0.5 M D-mannitol, $2 \mathrm{mM}$ 2-(N-morpholino) ethanesulfonic acid (MES; Sigma-Aldrich, cat. no. M3671), and $0.1 \times$ W5 medium, pH 5.7. The protoplasts were lysed with Passive Lysis Buffer (PLB, Promega) and assayed using the Dual-Luciferase Reporter Assay System (Promega). Relevant primer sequences are listed in Supplementary Data 5.

Subcellular localization. The entire coding sequence of $B O C 1$ was cloned into a GFP vector under the control of the $35 S$ promoter to generate $p 35 S:: B O C 1-G F P$. The RFP coding sequence was fused to the $\mathrm{C}$ terminus of OsMADS15 to generate OsMADS15-RFP and was used as a nuclear marker. The two constructs were transformed into protoplasts and incubated at $28{ }^{\circ} \mathrm{C}$ in the dark for $16 \mathrm{~h}$. Fluorescence was examined under a confocal laser scanning microscope.

Agrobacterium-mediated genetic transformation. The binary plasmid vectors described above were transferred into Agrobacterium tumefaciens strain EHA105. Sterilized mature seeds were inoculated on UINB medium and cultured at $28^{\circ} \mathrm{C}$ in the dark. After 7 days of incubating, the calli were collected and used for transformation. After 3 days of cocultivation with Agrobacterium at $25^{\circ} \mathrm{C}$ in constant darkness on NB-Acetosyringone (AS) medium (30 g/L sucrose, $10 \mathrm{~g} / \mathrm{L}$ glucose, $0.3 \mathrm{~g} / \mathrm{L}$ casamino acids, NB medium salt mixture [Duchefa Biochemie], $0.5 \mathrm{mg} / \mathrm{L}$ glutamine, and $2.5 \mathrm{mg} / \mathrm{L} 2,4-\mathrm{D}, 20 \mathrm{mg} / \mathrm{L} \mathrm{AS}, \mathrm{pH} 5.3$ ), the calli were washed two or three times with sterile water and rinsed once with sterile water containing $200 \mathrm{mg} / \mathrm{L}$ Timentin (Caisson Labs, USA) and $200 \mathrm{mg} / \mathrm{L}$ cephalosporin (Wako Pure Chemical Industries). The calli were cultured on UINB medium containing $50 \mathrm{mg} / \mathrm{L}$ hygromycin B (Hyg, Merck), $200 \mathrm{mg} / \mathrm{L}$ Timentin, and $200 \mathrm{mg} / \mathrm{L}$ cephalosporin for three rounds of 15 days each at $28^{\circ} \mathrm{C}$ in the dark. For regeneration, vigorously growing calli were transferred to MS regeneration medium $(30 \mathrm{~g} / \mathrm{L}$ sucrose, $30 \mathrm{~g} / \mathrm{L}$ sorbitol, $2 \mathrm{~g} / \mathrm{L}$ casamino acids, MS Medium Salt Mixture [Duchefa Biochemie], 1.1 g/L MES $2 \mathrm{mg} / \mathrm{L} 1$-naphthalene acetic acid, and $1 \mathrm{mg} / \mathrm{L}$ kinetin, $\mathrm{pH} 5.8$ ) and cultured for 15 to 30 days at $28^{\circ} \mathrm{C}(16 \mathrm{~h}$ light $/ 8 \mathrm{~h}$ dark). The means in the genetic transformation efficiency test represent data from 10 groups of independent experiments, with $\sim 80$ to $\sim 120$ transformed calli per group.

Quantitative determination of $\mathbf{H}_{2} \mathbf{O}_{2}$ contents and $\mathbf{H}_{2} \mathbf{O}_{2}$ flux. $\mathrm{H}_{2} \mathrm{O}_{2}$ concentrations were measured in calli subcultured for 21 days $^{63}$. The calli $(100 \mathrm{mg})$ were homogenized in $1 \mathrm{ml} 0.1 \%(\mathrm{w} / \mathrm{v})$ TCA in an ice bath. The homogenate was centrifuged at $12,000 \times g$ for $15 \mathrm{~min}$, and $1 \mathrm{ml}$ of the supernatant was added to $1 \mathrm{ml}$ $100 \mathrm{mM}$ potassium phosphate buffer ( $\mathrm{pH} 7.0)$ and $2 \mathrm{ml} 1 \mathrm{M} \mathrm{KI}$. The absorbance of the supernatant was read at $390 \mathrm{~nm}$ based on the fresh weight of the callus. $\mathrm{H}_{2} \mathrm{O}_{2}$ flux was measured using the Non-invasive Micro-test Technology (NMT) System (NMT100-SIM-XY, Younger USA Sci. \& Tech. Corp., Amherst, MA, USA) at the Xuyue Beijing NMT Service Center (Xuyue Beijing Sci. and Tech. Co., Ltd., Beijing China). Callus that had been subcultured for $21 \mathrm{~d}$ was fixed onto the bottom of a Petri using a filter paper strip and resin block. The callus in the Petri dish was incubated in measuring solution $(0.1 \mathrm{mM} \mathrm{KCl}, 0.1 \mathrm{mM} \mathrm{CaCl}, 0.1 \mathrm{mM} \mathrm{MgCl}$, $0.5 \mathrm{mM} \mathrm{NaCl}, 0.3 \mathrm{mM}$ MES, $0.2 \mathrm{mM} \mathrm{Na}_{2} \mathrm{SO} 4$, pH 6.5) for $20 \mathrm{~min}$. The measuring solution was discarded and replaced with $5 \sim 10 \mathrm{ml}$ of fresh measuring solution. $\mathrm{H}_{2} \mathrm{O}_{2}$ flux microsensor was placed a $\sim 10 \mu \mathrm{m}$ from the calli, and $\mathrm{H}_{2} \mathrm{O}_{2}$ flux at each site was measured for $10 \mathrm{~min}$; each measurement repeated four times. Flux data were obtained using imFluxes V2.0 software (Younger USA LLC, Amherst, MA 01002 , USA): a positive value represents efflux and a negative value represents influx. The $\mathrm{H}_{2} \mathrm{O}_{2}$ flux was calculated based on Fick's law of diffusion (5):

$$
J\left(\mathrm{pmol} / \mathrm{cm}^{2} / \mathrm{s}\right)=-D_{o} \times(d c / d x)
$$

where $J$ is the analyte $\mathrm{H}_{2} \mathrm{O}_{2}$ flux (unit: pmol.cm $\mathrm{cm}^{-2} \cdot \mathrm{s}^{-1}$ ), $d c$ is its concentration gradient, $d x$ is the distance between the two points $(30 \mu \mathrm{m})$, and $D_{0}$ is its diffusion constant (unit: $\mathrm{cm}^{-2} \cdot \mathrm{s}^{-1}$ ). 
Measurement of lipid peroxidation. MDA was measured using the thiobarbituric acid (TBA) assay ${ }^{64}$. The calli $(100 \mathrm{mg}$ ) were homogenized in $1 \mathrm{ml} 0.1 \%(\mathrm{w} / \mathrm{v})$ TCA in an ice bath. After centrifuging the homogenate at $12,000 \times g$ for $10 \mathrm{~min}, 0.5 \mathrm{ml}$ of the supernatant was combined with $0.5 \mathrm{ml} 0.6 \%$ thiobarbituric acid (TBA) and incubated at $95^{\circ} \mathrm{C}$ for $15 \mathrm{~min}$. The samples were cooled immediately in an ice bath and the absorbance of the supernatant measured at 450,532, and $600 \mathrm{~nm}$.

Assay of GSH content. GSH content was assayed by Shanghai Sanshu Biotechnology Co., Ltd (Shanghai, China). The reaction mixture contained plant extract, $0.1 \mathrm{M} \mathrm{NaH}_{2} \mathrm{PO}_{4}$ (pH 7.7), and DTNB (5,5-dithiobis-2-nitrobenzoic acid). Absorbance was recorded at $412 \mathrm{~nm}^{65}$.

Enzyme activity assays. The enzyme activity assays were carried out by Shanghai Sanshu Biotechnology Co., Ltd (Shanghai, China). Catalase (CAT) activity was measured based on the consumption of $\mathrm{H}_{2} \mathrm{O}_{2}$ and the decrease in absorbance at $240 \mathrm{~nm}^{66}$. Glutathione reductase (GR) was assayed based on the decrease in $A_{340}$ due to nicotinamide adenine dinucleotide phosphate (NADPH) oxidation. The reaction mixture of GR contained $1.0 \mathrm{ml}$ of $0.1 \mathrm{M}$ potassium phosphate buffer $(\mathrm{pH}$ 8.0) including $0.5 \mathrm{mM}$ EDTA, $0.5 \mathrm{mM} \mathrm{MgCl}_{2}, 1 \mathrm{mM} \mathrm{NADPH}, 10 \mathrm{mM}$ oxidized glutathione (GSSG), and $0.1 \mathrm{ml}$ enzyme extract ${ }^{67}$. The glutathione S-transferase (GST) was assayed using the substrate 1-chloro 2,4-dinitrobenzene (CDNB), and the activity was detected spectrophotometrically based on the change in absorbance at $340 \mathrm{~nm}^{68}$.

Endogenous hormone level measurements. The calli were ground into a powder in liquid nitrogen in a mortar and each sample $(0.05 \mathrm{~g})$ were transferred to a $1.5 \mathrm{ml}$ screw-cap tube. After adding $50 \mu \mathrm{l}$ of internal standards and $500 \mu \mathrm{l}$ of extraction solvent (2-propanol: $\mathrm{H}_{2} \mathrm{O}: \mathrm{HCl}=2: 1: 0.002$ ) to each tube, the sample was incubated on a shaker at $900 \times g$ for $30 \mathrm{~min}$ at $4{ }^{\circ} \mathrm{C}$. After adding $1 \mathrm{ml}$ trichloromethane, the sample was shaken for $30 \mathrm{~min}$ and centrifuged at $14,000 \times g$ for $5 \mathrm{~min}$ at $4{ }^{\circ} \mathrm{C}$ to form two phases. A $1.2 \mathrm{ml}$ sample of solvent from the lower phase was dried in a nitrogen evaporator, dissolved in $0.1 \mathrm{ml}$ methanol and endogenous SA levels quantified by high-performance liquid chromatography-mass spectrometry (HPLC-MS/MS) 69

Ethylene was analyzed according to a published method ${ }^{70}$ with minor modifications. The calli were placed into $100-\mathrm{mL}$ vials that were promptly sealed with a rubber plug and incubated at $28^{\circ} \mathrm{C}$ for $48 \mathrm{~h}$ in the dark. Ethylene content in $2 \mathrm{ml}$ of the headspace gas was analyzed with a gas chromatograph (GC-17A, Shimadzu, Japan).

RNA-seq analysis. Total RNA was isolated from Teqing and YIL25, pCPL and Teqing, and pOE and Teqing calli (subcultured for 21 days) with three biological replicates. Paired-end libraries were constructed and sequenced on the Illumina HiSeq 2500 platform at the Novogene Company (China). The RNA-seq reads were mapped to the reference genome (Os-Nipponbare-Refrence-IRGSP-1.0, MSU7) using TopHat2 with default parameters, and the FPKM (fragments per kilobase of exon per million mapped reads) of each gene and the DEGs (fold change $\geq 2$, FDR $<0.001)$ between YIL25 and Teqing, pCPL and Teqing, and pOE and Teqing were calculated $^{71}$

Sequencing and data analysis. The $\sim 4.6 \mathrm{~kb}$ genomic fragment covering the entire BOC1 gene ( $2740 \mathrm{bp}$ ), the $1,452 \mathrm{bp} 5^{\prime}$-flanking region, and the $380 \mathrm{bp} 3^{\prime}$-flanking region were amplified using five PCR primer pairs and sequenced using the Sanger sequencing approach. A previously published dataset in 1,083 diverse rice accessions was used to calculate the fixation index $\left(F_{\mathrm{ST}}\right)$ across chromosome 3 between indica and japonica varieties using VCFtools 0.1 .15 , with a $100-\mathrm{Kb}$ window $\operatorname{size}^{36,72}$

Data analysis. A two-tailed Student's $t$-test was performed using SPSS version 16 (SPSS Inc., Chicago, IL, USA). QTL analysis was performed using MapManager QTXb $20^{73}$. Amino acid sequences homologous to BOC1 were downloaded from the National Center for Biotechnology Information website (http://blast.ncbi.nlm. nih.gov/). Phylogenetic trees were constructed using the neighbor-joining algorithm in MEGA version 7 with 1000 bootstrap replications ${ }^{74}$. The association tests were analyzed by $\mathrm{R}$ v3.6.1.

Reporting summary. Further information on research design is available in the Nature Research Reporting Summary linked to this article.

\section{Data availability}

Data supporting the findings of this work are available within the paper and its Supplementary Information files. A reporting summary for this Article is available as a Supplementary Information file. The datasets generated and analyzed during the current study are available from the corresponding author upon request. RNA-seq data for callus that had been subcultured for 21 days from Teqing and YIL25, Teqing and the complementation line pCPL-BOC1-Teqing, and Teqing and the overexpression line pOE-BOC1-Teqing was deposited in the National Center for Biotechnology Information
(NCBI) under GEO accession number GSE142094. The source data underlying Figs. $1 \mathrm{j}$, $1 \mathrm{k}, 2 \mathrm{a}, 2 \mathrm{~b}, 3 \mathrm{e}, 3 \mathrm{j}, 3 \mathrm{o}, 4 \mathrm{a}, 4 \mathrm{c}, 5,6,8 \mathrm{~b}, 8 \mathrm{c}, 8 \mathrm{e}$, and $8 \mathrm{f}$, as well as Supplementary Figs. 2, 5, 7, $8 \mathrm{k}$, and 9 are provided as a Source Data file.

Received: 29 March 2019; Accepted: 20 December 2019; Published online: 23 January 2020

\section{References}

1. He, Y. et al. Changes in morphology and biochemical indices in browning callus derived from Jatropha curcas hypocotyls. Plant Cell Tiss. Org. 98, 11-17 (2009).

2. Tóth, K., Haapala, T. \& Hohtola, A. Alleviation of browning in oak explants by chemical pretreatments. Biol. Plant. 36, 511-517 (1994).

3. Krishna, H. et al. Mango explant browning: effect of ontogenic age, mycorrhization and pre-treatments. Sci. Hortic. 118, 132-138 (2008)

4. Uchendu, E. E., Paliyath, G., Brown, D. C. W. \& Saxena, P. K. In vitro propagation of North American ginseng (Panax quinquefolius L.). Vitr. Cell Dev.-Plant. 47, 710-718 (2011).

5. Thomas, T. D. The role of activated charcoal in plant tissue culture. Biotechnol. Adv. 26, 618-631 (2008).

6. Han, M. L., Gleave, A. P. \& Wang, T. C. Efficient transformation of Actinidia arguta by reducing the strength of basal salts in the medium to alleviate callus browning. Plant Biotechnol. Rep. 4, 129-138 (2010).

7. Dong, Y. S. et al. Mechanisms and effective control of physiological browning phenomena in plant cell cultures. Physiol. Plant. 156, 13-28 (2016).

8. Mayer, A. M. \& Harel, E. Polyphenol oxidases in plant. Phytochemistry 18, 193-215 (1979).

9. Murata, M. et al. A transgenic apple callus showing reduced polyphenol oxidase activity and lower browning potential. Biosci. Biotechnol. Biochem. 65, 383-388 (2001).

10. Tang, W. \& Newton, R. J. Increase of polyphenol oxidase and decrease of polyamines correlate with tissue browning in Virginia pine (Pinus virginiana Mill.). Plant Sci. 167, 621-628 (2004).

11. Sarmadi, M. et al. The effects of salicylic acid and glucose on biochemical traits and taxane production in a Taxus baccata callus culture. Plant Physiol. Bioch. 132, 271-280 (2018).

12. Adkins, S. W., Shiraishi, T. \& Mccomb, J. A. Rice callus physiologyidentification of volatile emissions and their effects on culture growth. Physiol. Plant. 78, 526-531 (1990).

13. Kobayashi, Y., Fukui, H. \& Tabata, M. Effect of carbon dioxide and ethylene on berberine production and cell browning in Thalictrum minus cell cultures. Plant Cell Rep. 9, 496-499 (1991).

14. Ogawa, T., Fukuoka, H., Yano, H. \& Ohkawa, Y. Relationships between nitrite reductase activity and genotype-dependent callus growth in rice cell cultures. Plant Cell Rep. 18, 576-581 (1999).

15. Abe, T. \& Futsufara, Y. Genotypic variability for callus formation and plant regeneration in rice (Oryza sativa L.). Theor. Appl. Genet. 72, 3-10 (1986).

16. Taguchi-Shiobara, F. et al. Mapping quantitative trait loci associated with regeneration ability of seed callus in rice, Oryza sativa L. Theor. Appl. Genet. 95, 823-833 (1997).

17. Taguchi-Shiobara, F. et al. Mapping QTLs that control the performance of rice tissue culture and evaluation of derived near-isogenic lines. Theor. Appl. Genet. 112, 968-976 (2006).

18. Takeuchi, Y., Abe, T. \& Sasahara, T. RFLP mapping of QTLs influencing shoot regeneration from mature seed-derived calli in rice. Crop Sci. 40, 245-247 (2000).

19. Nishimura, A. et al. Isolation of a rice regeneration quantitative trait loci gene and its application to transformation systems. Proc. Natl Acad. Sci. USA 102, 11940-11944 (2005).

20. Ozawa, K. \& Kawahigashi, H. Positional cloning of the nitrite reductase gene associated with good growth and regeneration ability of calli and establishment of a new selection system for Agrobacterium-mediated transformation in rice (Oryza sativa L.). Plant Sci. 170, 384-393 (2006).

21. Li, Z. et al. A single genetic locus in chromosome 1 controls conditional browning during the induction of calli from mature seeds of Oryza sativa ssp indica. Plant Cell Tiss. Org. 89, 237-245 (2007).

22. Zhao, L. N. et al. Identification of quantitative trait loci controlling rice mature seed culturability using chromosomal segment substitution lines. Plant Cell Rep. 28, 247-256 (2009).

23. $\mathrm{Li}, \mathrm{S}$. J. et al. Identification of QTLs associated with tissue culture response through sequencing-based genotyping of RILs derived from 93-11 x Nipponbare in rice (Oryza sativa). Plant Cell Rep. 32, 103-116 (2013).

24. Zhang, K. et al. Quantitative trait loci for mature embryo culturability traits from Yuanjiang common wild rice (Oryza rufipogon Griff.). Indian J. Genet. Pl. Br. 76, 167-172 (2016). 
25. Yanagisawa, S. \& Schmidt, R. J. Diversity and similarity among recognition sequences of Dof transcription factors. Plant J. 17, 209-214 (1999).

26. Abe, H. et al. Arabidopsis AtMYC2 (bHLH) and AtMYB2 (MYB) function as transcriptional activators in abscisic acid signaling. Plant Cell 15, 63-78 (2003).

27. Chinnusamy, V. et al. ICE1: a regulator of cold-induced transcriptome and freezing tolerance in Arabidopsis. Gene. Dev. 17, 1043-1054 (2003)

28. Simpson, S. D. et al. Two different novel cis-acting elements of erd1, a $\operatorname{clp} A$ homologous Arabidopsis gene function in induction by dehydration stress and dark-induced senescence. Plant J. 33, 259-270 (2003).

29. Zhu, J. K. Abiotic stress signaling and responses in plants. Cell 167, 313-324 (2016).

30. Liu, Y. et al. $A B A$ overly-sensitive 5 ( $A B O 5$ ), encoding a pentatricopeptide repeat protein required for cis-splicing of mitochondrial nad2 intron 3, is involved in the abscisic acid response in Arabidopsis. Plant J. 63, 749-765 (2010).

31. Singh, S. et al. Down-regulation of OsSAG12-1 results in enhanced senescence and pathogen-induced cell death in transgenic rice plants. J. Biosci. 38, 583-592 (2013).

32. Zangar, R. C., Davydov, D. R. \& Verma, S. Mechanisms that regulate production of reactive oxygen species by cytochrome P450. Toxicol. Appl. Pharm. 199, 316-331 (2004).

33. Niu, N. N. et al. EAT1 promotes tapetal cell death by regulating aspartic proteases during male reproductive development in rice. Nat. Commun. $4, \mathrm{X}$ (2013).

34. Jing, S. J., Zhou, X., Song, Y. \& Yu, D. Q. Heterologous expression of OsWRKY23 gene enhances pathogen defense and dark-induced leaf senescence in Arabidopsis. Plant Growth Regul. 58, 181-190 (2009).

35. Kim, S. G. et al. The RNase activity of rice probenazole-induced protein 1 (PBZ1) plays a key role in cell death in plants. Mol. Cells 31, 25-31 (2011).

36. Huang, X. H. et al. A map of rice genome variation reveals the origin of cultivated rice. Nature 490, 497-501 (2012).

37. You, J. et al. The SNAC1-targeted gene OsSRO1c modulates stomatal closure and oxidative stress tolerance by regulating hydrogen peroxide in rice. J. Exp. Bot. 64, 569-583 (2013).

38. Overmyer, K. et al. Ozone-sensitive Arabidopsis rcd1 mutant reveals opposite roles for ethylene and jasmonate signaling pathways in regulating superoxidedependent cell death. Plant Cell 12, 1849-1862 (2000).

39. Overmyer, K. et al. Ozone-induced programmed cell death in the Arabidopsis radical-induced cell death1 mutant. Plant Physiol. 137, 1092-1104 (2005).

40. Ahlfors, R. et al. Arabidopsis radical-induced cell death1 belongs to the WWE protein-protein interaction domain protein family and modulates abscisic acid, ethylene, and methyl jasmonate responses. Plant Cell 16, 1925-1937 (2004).

41. Ahlfors, R., Brosché, M., Kollist, H. \& Kangasjärvi, J. Nitric oxide modulates ozone-induced cell death, hormone biosynthesis and gene expression in Arabidopsis thaliana. Plant J. 58, 1-16 (2009).

42. Katiyar-Agarwal, S. et al. The plasma membrane $\mathrm{Na}^{+} / \mathrm{H}^{+}$antiporter SOS1 interacts with RCD1 and functions in oxidative stress tolerance inArabidopsis. Proc. Natl Acad. Sci. USA 103, 18816-18821 (2006).

43. Hiltscher, $\mathrm{H}$. et al. The radical induced cell death protein 1 (RCD1) supports transcriptional activation of genes for chloroplast antioxidant enzymes. Front. Plant Sci. 5, 475 (2014).

44. Niu, X. M. et al. Transposable elements drive rapid phenotypic variation in Capsella rubella. Proc. Natl Acad. Sci. USA 116, 6908-6913 (2019).

45. Xia, C. et al. A TRIM insertion in the promoter of $M s 2$ causes male sterility in wheat. Nat. Commun. 8, 15407 (2017).

46. Mao, H. D. et al. A transposable element in a NAC gene is associated with drought tolerance in maize seedlings. Nat. Commun. 6, 8326 (2015).

47. Jones, A. M. P. \& Saxena, P. K. Inhibition of phenylpropanoid biosynthesis in Artemisia annua L.: a novel approach to reduce oxidative browning in plant tissue culture. PLoS One 8, e76802 (2013).

48. Gechev, T. S. et al. Reactive oxygen species as signals that modulate plant stress responses and programmed cell death. Bioessays 28, 1091-1101 (2006).

49. Overmyer, K., Brosché, M. \& Kangasjärvi, J. Reactive oxygen species and hormonal control of cell death. Trends Plant Sci. 8, 335-342 (2003).

50. Kangasjärvi, S. et al. Photosynthesis, photorespiration, and light signalling in defence responses. J. Exp. Bot. 63, 1619-1636 (2012).

51. Petrov, V., Hille, J., Mueller-Roeber, B. \& Gechev, T. S. ROS-mediated abiotic stress-induced programmed cell death in plants. Front. Plant Sci. 6, 69 (2015).

52. Pan, D. J. et al. Interactions between salicylic acid and antioxidant enzymes tilting the balance of $\mathrm{H}_{2} \mathrm{O}_{2}$ from photorespiration in non-target crops under halosulfuron-methyl stress. Pestic. Biochem. Phys. 143, 214-223 (2017)

53. Chao, Y. Y. et al. Salicylic acid-mediated hydrogen peroxide accumulation and protection against $\mathrm{Cd}$ toxicity in rice leaves. Plant Soil. 329, 327-337 (2010).
54. Housti, F., Coupe, M. \& Dauzac, J. Effect of ethylene on enzymatic activities involved in the browning of Hevea brasiliensis callus. Physiol. Plant. 86, 445-450 (1992)

55. Shapiguzov, A. et al. Arabidopsis RCD1 coordinates chloroplast and mitochondrial functions through interaction with ANAC transcription factors. eLife 8, e43284 (2019).

56. Sun, C. Q. et al. Comparison of the genetic diversity of common wild rice (Oryza rufipogon Griff.) and cultivated rice (O. sativa L.) using RFLP markers. Theor. Appl. Genet. 102, 157-162 (2001).

57. Tan, L. B. et al. Development of Oryza rufipogon and O. sativa introgression lines and assessment for yield related quantitative trait loci. J. Integr. Plant Biol. 49, 871-884 (2007).

58. Chu, C. C. et al. Establishment of an efficient medium for anther culture of rice through comparative experiments on nitrogen sources. Sci. China Chem. 18, 659-668 (1975).

59. Gamborg, O. L., Miller, R. A. \& Ojima, K. Nutrient requirements of suspension cultures of soybean root cells. Exp. Cell Res. 50, 151-158 (1968).

60. Livak, K. J. \& Schmittgen, T. D. Analysis of relative gene expression data using real-time quantitative PCR and the $2-\triangle \triangle \mathrm{CT}$ method. Methods 25, 402-408 (2001).

61. Javelle, M., Marco, C. F. \& Timmermans, M. In situ hybridization for the precise localization of transcripts in plants. J. Vis. Exp. 57, e3328 (2011).

62. Shen, J. et al. Isolation, culture, and transient transformation of plant protoplasts. Curr. Protoc. Cell Biol. 63, 1-17 (2014).

63. Velikova, V., Yordanov, I. \& Edreva, A. Oxidative stress and some antioxidant systems in acid rain-treated bean plants: protective role of exogenous polyamines. Plant Sci. 151, 59-66 (2000).

64. Peever, T. L. \& Higgins, V. J. Electrolyte leakage, lipoxygenase, and lipidperoxidation induced in tomato leaf tissue by specific and nonspecific elicitors from Cladosporium fulvum. Plant Physiol. 90, 867-875 (1989).

65. Griffith, O. W. Determination of glutathione and glutathione disulfide using glutathione-reductase and 2-vinylpyridine. Anal. Biochem. 106, 207-212 (1980).

66. Jiménez, A., Hernández, J. A., DelRio, L. A. \& Sevilla, F. Evidence for the presence of the ascorbate-glutathione cycle in mitochondria and peroxisomes of pea leaves. Plant Physiol. 114, 275-284 (1997).

67. Edwards, E. A., Rawsthorne, S. \& Mullineaux, P. M. Subcellular distribution of multiple forms of glutathione reduetase in leaves of pea (Pisum sativum L.). Planta 180, 278-284 (1990).

68. Habig, W. H. \& Jakoby, W. B. Assay for differentiation of glutathione Stransferases. Method Enzymol. 77, 398-405 (1981).

69. Pan, X., Welti, R. \& Wang, X. Quantitative analysis of major plant hormones in crude plant extracts by high-performance liquid chromatography-mass spectrometry. Nat. Protoc. 5, 986-992 (2010).

70. Roustan, J. P., Latche, A. \& Fallot, J. Inhibition of ethylene production and stimulation of carrot somatic embryogenesis by salicylic acid. Biol. Plant. 32, 273-276 (1990).

71. Trapnell, C. et al. Transcript assembly and quantification by RNA-Seq reveals unannotated transcripts and isoform switching during cell differentiation. Nat. Biotechnol. 28, 511-515 (2010).

72. Danecek, P. et al. The variant call format and VCFtools. Bioinformatics 27, 2156-2158 (2011).

73. Manly, K. F., Cudmore, R. J. \& Meer, J. M. Map Manager QTX, cross-platform software for genetic mapping. Mamm. Genome 12, 930-932 (2001).

74. Kumar, S., Stecher, G. \& Tamura, K. MEGA7: molecular evolutionary genetics analysis version 7.0 for bigger datasets. Mol. Biol. Evol. 33, 1870-1874 (2016).

\section{Acknowledgements}

We thank the International Rice Research Institute, Chinese Rice Research Institute, Institute of Crop Sciences of Chinese Academy of Agricultural Sciences, Guangxi Academy of Agricultural Sciences, and Guangdong Academy of Agricultural Sciences for providing the wild and cultivated rice germplasm. This project was supported by the National Natural Science Foundation of China (grant number 31571626) and the National Transgenic Major Project of China (grant number 2016ZX08001-004).

\section{Author contributions}

Y.F. conceived and designed the research. K.Z. performed the experiments, analyzed data, and wrote the paper. J.S., M.X., Z.Z. and J.H. assisted with experiments. X.Z. and X.M helped analyze the data. C.L and Y.L. helped collect the O. rufipogon accessions. L.T., Z.Z., H.C., F.L., H.S., P.G., W.Z. and C.S. provided technical assistance and revised the paper. Y.F. performed data analysis and revised the paper.

\section{Competing interests}

The authors declare no competing interests. 


\section{Additional information}

Supplementary information is available for this paper at https://doi.org/10.1038/s41467019-14265-0

Correspondence and requests for materials should be addressed to Y.F.

Peer review information Nature Communications thanks Kirk Overmyer, and the other, anonymous, reviewer(s) for their contribution to the peer review of this work. Peer reviewer reports are available.

Reprints and permission information is available at http://www.nature.com/reprints

Publisher's note Springer Nature remains neutral with regard to jurisdictional claims in published maps and institutional affiliations. (c) (i) Open Access This article is licensed under a Creative Commons Attribution 4.0 International License, which permits use, sharing, adaptation, distribution and reproduction in any medium or format, as long as you give appropriate credit to the original author(s) and the source, provide a link to the Creative Commons license, and indicate if changes were made. The images or other third party material in this article are included in the article's Creative Commons license, unless indicated otherwise in a credit line to the material. If material is not included in the article's Creative Commons license and your intended use is not permitted by statutory regulation or exceeds the permitted use, you will need to obtain permission directly from the copyright holder. To view a copy of this license, visit http://creativecommons.org/ licenses/by/4.0/.

(C) The Author(s) 2020 